\title{
Correlation of $\gamma$-ray and high-energy cosmic ray fluxes from the giant lobes of Centaurus A
}

\author{
N. Fraija ${ }^{1}$ \\ Instituto de Astronomía, UNAM, México, 04510 \\ nifraijaeastro.unam.mx
}

March 28, 2021

Received __ ; accepted _ _

\footnotetext{
${ }^{1}$ Luc Binette-Fundación UNAM Fellow. Instituto de Astronomía, Universidad Nacional Autónoma de México, Circuito Exterior, C.U., A. Postal 70-264, 04510 México D.F., México
} 


\begin{abstract}
The spectral energy distribution of giant lobes shows one main peak detected by the Wilkinson Microwave Anisotropy Probe at low energy of $10^{-5} \mathrm{eV}$ and a faint $\gamma$-ray flux imaged by Fermi Large Area Telescope at energy $\geq 100 \mathrm{MeV}$. On the other hand, Pierre Auger Observatory associated some ultra-high-energy cosmic rays with the direction of Centaurus A and IceCube reported 28 neutrino-induced events in a TeV $\mathrm{PeV}$ energy range, although none of them related with this direction. In this work we describe the spectra for each of the lobes, the main peak with synchrotron radiation, and the high-energy emission with $p p$ interactions. Obtaining a good description of the main peak, we deduce the magnetic fields, electron densities and the age of the lobes. Describing successfully the $\gamma$-ray emission by pp interactions and considering as targets those thermal particles in the lobes with density in the range $10^{-10}$ to $10^{-4} \mathrm{~cm}^{-3}$, we calculate the number of ultra-high-energy cosmic rays. Although $\gamma$-spectrum is well described with any density in the range, only when $10^{-4} \mathrm{~cm}^{-3}$ is considered, the expected number of events is very similar to that observed by Pierre Auger Observatory, otherwise we obtain an excessive luminosity. In addition, correlating the $\gamma$-ray and neutrino fluxes through pp interactions we calculate the number of high-energy neutrinos expected in IceCube. Our analysis indicates that neutrinos above $1 \mathrm{TeV}$ cannot be produced in the lobes of Centaurus A, which is consistent with the results recently published by IceCube Collaboration.
\end{abstract}

Subject headings: Galaxies: active - Galaxies: individual (Centaurus A) - Physical data and processes: acceleration of particles - Physical data and processes: radiation mechanism: nonthermal 


\section{Introduction}

Centaurus A (Cen A), at a distance of $3.8 \mathrm{Mpc}$, is the nearest radio-loud active galactic nucleus (AGN). Due to its distance, Cen A is an excellent source for studying the physics of relativistic outflows and radio lobes. It has a jet with an axis subtending an angle to the line of sight estimated as $15^{\circ}-80^{\circ}$ (see, e.g. Horiuchi et al. 2006, and reference therein) and two giant lobes oriented primarily in the north-south direction, which subtend $\sim 10^{\circ}$ on the sky. They were imaged and analyzed by Parkes radio telescope at $6.3 \mathrm{~cm}$ (Junkes et al. 1993; Alvarez et al. 2000) and at 22, 33,41,61 and $94 \mathrm{GHz}$ by the Wilkinson Microwave Anisotropy Probe (WMAP; Hinshaw \& et al. 2009; Page \& et al. 2003; Hardcastle et al. 2009; Abdo \& et al. 2010a). Also for a period of 10 months, Cen A was monitored by Large Area Telescope (LAT) on board the Fermi Gamma-Ray Space Telescope (Atwood et al. 2009) and $\gamma$-ray excesses were detected from both lobes. The resulting LAT image showed the $\gamma$-ray peak coincident with the active galactic nucleus detected by the Compton/EGRET instrument (Hartman et al.|1999). Assuming a power law for the $\gamma$-ray spectra and from the resultant test statistics (Mattox et al. 1996) LAT recorded a flux of $\left[0.77(+0.23 /-0.19)_{\text {stat }}( \pm 0.39)_{\text {syst }}\right] \times 10^{-7} \mathrm{ph} \mathrm{cm}^{-2} \mathrm{~s}^{-1}$ with photon index $2.52(+0.16 /-0.19)_{\text {stat }}( \pm 0.25)_{\text {syst }}$ for the north lobe and a flux of $[1.09(+0.24 /-$

$\left.0.21)_{\text {stat }}( \pm 0.32)_{\text {syst }}\right] \times 10^{-7} \mathrm{ph} \mathrm{cm}^{-2} \mathrm{~s}^{-1}$ with photon index $2.60(+0.14 /-0.15)_{\text {stat }}( \pm 0.20)_{\text {syst }}$ for the south lobe (Abdo \& et al. 2010a).

Hardcastle et al. (2006) have claimed that the oncoming jet enters the northern inner lobe, encrusted in the thermal interstellar gas of NGC5128, at $\sim 3.5 \mathrm{kpc}$. Based on deep Chandra observations, Hardcastle et al. (2007) reclaimed that the receding jet extends out to $\sim 2.5 \mathrm{kpc}$ in protection in $\mathrm{X}$ rays, showing also up on a similar scale in radio (Hardcastle et al. 2003; Tingav et al. (1998). Based on the detection of extended thermal X-ray emission from this region, Kraft et al. (2009) interpreted the northern middle lobe as an old structure that has recently become reconnected to the energy supply from the jet (Wykes et al. 2013).

Based on X-ray (0.5-2.5 keV) measurements and supposing that all the emission comes from 
a uniform thermal plasma, Hardcastle et al. (2009) established an strict upper limit on this plasma, that is $n_{p} \sim 10^{-4} \mathrm{~cm}^{-3}$. Recently, considering the internal Faraday rotation scenario, O'Sullivan et al. (2013) presented a positive detection of the internal depolarization signal leading to the same value of density. Also, Stawarz et al. (2013) presenting an analysis of the diffuse X-ray emission found a tentative detection of a soft excess component with an energy of $k T \sim 0.5 \mathrm{keV}$, corresponding to the same value of the number density of the thermal gas. However, Wykes et al. (2013) estimating the values of total entrainment, buoyancy age and the average volume of the giant lobes found a different number density of thermal particles $n_{p} \sim 10^{-9} \mathrm{~cm}^{-3}$. In addition, they calculated that the relativistic electron number densities for four giant lobe sectors defined by Hardcastle et al. (2009) were in the range $1.0 \times 10^{-11} \mathrm{~cm}^{-3} \leq N_{e} \leq 1.5 \times 10^{-8} \mathrm{~cm}^{-3}$.

Otherwise, based on the report given by Pierre Auger Collaboration (PAO) with respect to the anisotropy in the arrival direction of ultra-high-energy cosmic rays (UHECRs) (Pierre Auger Collaboration \& et al. 2007, 2008) and the possible correlation with Cen A, some authors have pointed out that Cen A has the potential to accelerate protons up to ultra-high energies (e.g. Gorbunov et al. 2008; Moskalenko et al. 2009; Dermer et al.(2009).

Recently, IceCube reported the detection of events in an energy range of $\mathrm{TeV}-\mathrm{PeV}$ (IceCube Collaboration et al. 2013a, b) and although these events have been discussed to have an extragalactic origin(Cholis \& Hooper 2012; Liu \& Wang 2013; Murase \& Ioka 2013; Razzaque 2013; Fraija 2013), they were not correlated with the direction of Cen A.

On the other hand, although energy ranges in radio, infrared, optical (Winkler \& White 1975; Mushotzky et al. 1976; Bowver et al. 1970; Baity \& et al. 1981), X-ray and $\gamma$-rays (MeV-TeV) (Abdo \& et al. 2010b; Sreekumar et al. 1999; Aharonian \& et al. 2009) have been detected close to the core of Cen A, only photons in radio and $\gamma$-rays have been collected from the lobes and, while the spectral energy distribution (SED) of each lobe has been described with leptonic models; Radio (WMAP) data through synchrotron radiation and Fermi-LAT data through inverse Compton-scattered (IC) radiation from the cosmic microwave background (CMB) 
(Crusius \& Schlickeiser 1986; Blumenthal \& Gould 1970) and extragalactic background light (EBL)(Abdo \& et al. 2010a; Hardcastle et al. 2009; Yang et al. 2012; Hardcastle et al. 2009; Hauser \& Dwek 2001; Georganopoulos et al. 2008; Raue \& Mazin 2008), the SED near the core has been successfully described through synchrotron self-Compton (SSC), and leptonic and hadronic models; SSC to fit the two main peaks (Abdo \& et al. 2010b; Chiaberge et al. 2001; Lenain et al. 2008) and leptonic (Hardcastle \& Croston 2011) and hadronic models to explain the flux at TeV energies (Dermer et al. 2009; Fraija et al. 2012; Sahu et al. 2012). In addition, these authors extrapolating the hadronic model ( $\mathrm{pp}$ or $\mathrm{p} \gamma$ interactions) to energies of $\sim 10^{20} \mathrm{eV}$, have correlated the number of UHECRs observed by PAO with the flux in TeV energy range. Also, based on these observations, Cuoco \& Hannestad (2008) and Halzen \& O'Murchadha (2008) have forecasted the expected rate of HE neutrinos in IceCube.

In this work we develop a leptonic and hadronic model to describe the SED for each of the lobes. For the leptonic model, we present the synchrotron emission to fit WMAP data and for the hadronic model, we introduce pp interactions to fit Fermi-LAT data. Also other mechanisms of emission (IC scattering of CMB and EBL as well as $\mathrm{p} \gamma$ interactions) are discussed. Correlating the $\gamma$-ray, UHECR and neutrino fluxes through pp interactions, we extend the proton and neutrino spectra through a simple and broken power law up to energy ranges of PAO and IceCube, respectively to estimate the number of expected events in each of the experiments.

\section{Emission Processes from Lobes}

Detections of non-thermal radiation and soft X-rays from lobes have pointed out that they could be filled with a magnetized plasma and thermal particles (Hardcastle et al. 2009; O'Sullivan et al. 2013; Wykes et al. 2013; Stawarz et al. 2013; Abdo \& et al. 2010a). Based on this approach we are going to develop synchrotron radiation as leptonic process and pp interactions as hadronic process to describe the whole energy range of the lobes. 


\subsection{Synchrotron Radiation}

The non-thermal radio emission can be inferred through synchrotron radiation generated by an electron distribution. The population of these accelerated electrons can be described by a broken power-law given by (Longair 1994; Hardcastle et al. 2001, 2006; Hardcastle \& Croston 2011)

$$
N_{e}\left(\gamma_{e}\right)=A_{e} \begin{cases}\gamma_{e}^{-\alpha} & \gamma_{e, m}<\gamma_{e}<\gamma_{e, b}, \\ \gamma_{e, b} \gamma_{e}^{-(\alpha+1)} & \gamma_{e, b} \leq \gamma_{e}<\gamma_{e, \max },\end{cases}
$$

where $A_{e}$ is the proportionality electron constant, $\alpha$ is the spectral index and $\gamma_{e, i}$ are the electron Lorentz factors. The index $i$ is $\mathrm{m}, \mathrm{b}$ or max for minimum, break and maximum, respectively. Assuming an equipartition of energy density between magnetic field $U_{B}=B^{2} / 8 \pi$ and electrons $U_{e}=m_{e} \int \gamma_{e} N_{e}\left(\gamma_{e}\right) d \gamma_{e}$, the electron Lorentz factors are

$$
\begin{aligned}
\gamma_{e, m} & =\frac{(\alpha-2)}{m_{e}(\alpha-1)} \frac{U_{e}}{N_{e}} \\
\gamma_{e, b} & =\frac{3 m_{e}}{4 \sigma_{T} \beta^{2}} U_{B}^{-1} t_{s y n}^{-1} \\
\gamma_{e, \max } & =\left(\frac{9 q_{e}^{2}}{8 \pi \sigma_{T}^{2} \beta^{4}}\right)^{1 / 4} U_{B}^{-1 / 4}
\end{aligned}
$$

where the constants $m_{p}, m_{e}, q_{e}$ and $\sigma_{T}$ are the proton and electron mass, the electric charge and Thomson cross section, respectively, $\beta=v / c \sim 1$ and $z=0.00183$ is the redshift(Israel 1998). The observed photon energies, $\epsilon_{\gamma}^{o b s}\left(\gamma_{e}\right)=\sqrt{\frac{8 \pi q_{e}^{2}}{m_{e}^{2}}}(1+z)^{-1} \delta_{D} U_{B}^{1 / 2} \gamma_{e, i}^{2}$, for each Lorentz factor (eq. 2) are

$$
\begin{aligned}
\epsilon_{\gamma, m}^{o b s} & =\frac{\sqrt{8 \pi} q_{e}(\alpha-2)^{2}}{m_{e}^{3}(\alpha-1)^{2}}(1+z)^{-1} \delta_{D} U_{B}^{1 / 2} U_{e}^{2} N_{e}^{-2}, \\
\epsilon_{\gamma, c}^{o b s} & =\frac{9 \sqrt{2 \pi} q_{e} m_{e}}{8 \sigma_{T}^{2} \beta^{4}}(1+z)^{-1} \delta_{D} U_{B}^{-3 / 2} t_{s y n}^{-2}, \\
\epsilon_{\gamma, \text { max }}^{o b s} & =\frac{3 q_{e}^{2}}{m_{e} \sigma_{T} \beta^{2}}(1+z)^{-1} \delta_{D},
\end{aligned}
$$

where we have applied the synchrotron cooling time scale,

$$
t_{\text {syn }}=\frac{E_{e}^{\prime}}{\left(d E_{e} / d t\right)^{\prime}}=\frac{3 m_{e}^{2}}{4 \sigma_{T} \beta^{2}} U_{B}^{-1} E_{e}^{\prime-1}
$$


and $\delta_{D}$ is the Doppler factor. On the other hand, the synchrotron spectrum is obtained by the shape of the electron spectrum (eq. 1) rather than the emission spectrum of a single particle. Therefore, the energy radiated in the range $\epsilon_{\gamma}$ to $\epsilon_{\gamma}+d \epsilon_{\gamma}$ is given by electrons between $E_{e}$ and $E_{e}+d E_{e}$; then we can estimate the photon spectrum through emissivity $\epsilon_{\gamma} N_{\gamma}\left(\epsilon_{\gamma}\right) d \epsilon_{\gamma}=\left(-\frac{d E_{e}}{d t}\right) N_{e}\left(E_{e}\right) d E_{e}$. Following Longair (1994) and Rybicki \& Lightman (1986), it is easy to show that if electron distribution has spectral indexes $\alpha$ and $(\alpha-1)$, then the photon distribution has spectral indexes $p=(\alpha-1) / 2$ and $p=\alpha / 2$, respectively. The proportionality constant is estimated calculating the total number of radiating electrons in the actual volume, $n_{e}=N_{e} / V=4 \pi N_{e} r_{d}^{3} / 3$, the maximum radiation power $P_{\nu, \text { max }}^{o b s} \simeq \frac{d E / d t}{\epsilon_{\gamma}\left(\gamma_{e}\right)}$ and the distance $\mathrm{D}_{z}$ from the source. Then, we can obtain the observed synchrotron spectrum as follow

$$
\epsilon_{\gamma}^{2} N_{\gamma}\left(\epsilon_{\gamma}\right)=A_{s y n, \gamma} \begin{cases}\left(\frac{\epsilon_{\gamma}}{\epsilon_{\gamma, m}}\right)^{4 / 3} & \epsilon_{\gamma}^{o b s}<\epsilon_{\gamma, m}^{o b s}, \\ \left(\frac{\epsilon_{\gamma}}{\epsilon_{\gamma, m}}\right)^{-(\alpha-3) / 2} & \epsilon_{\gamma, m}^{o b s}<\epsilon_{\gamma}^{o b s}<\epsilon_{\gamma, c}^{o b s}, \\ \left(\frac{\epsilon_{\gamma, c}}{\epsilon_{\gamma, m}}\right)^{-(\alpha-3) / 2}\left(\frac{\epsilon_{\gamma}}{\epsilon_{\gamma, c}}\right)^{-(\alpha-2) / 2}, & \epsilon_{\gamma, c}^{o b s}<\epsilon_{\gamma}^{o b s}<\epsilon_{\gamma, \max }^{o b s}\end{cases}
$$

where

$$
A_{s y n, \gamma}=\frac{P_{\nu, \max }^{o b s} n_{e}}{4 \pi D_{z}^{2}} \epsilon_{\gamma, m}^{o b s} \simeq \frac{8 \pi \sigma_{T} \beta^{2}(\alpha-2)^{2}}{9 m_{e}^{2}(\alpha-1)^{2} D_{z}^{2}}(1+z)^{-2} \delta_{D}^{2} U_{B} U_{e}^{2} N_{e} r_{d}^{3}
$$

It is important to clarify that $r_{d}$ is the region where emitting electrons are confined. Eq. 5] represents the peak at lower energies (radio wavelength) of the SED for each of the lobes.

\section{2. $P P$ interactions}

We suppose that accelerated protons are cooled down through pp interactions (Becker 2008; Atoyan \& Dermer 2003; Dermer \& Menon 2009; Aharonian 2002). Pp interactions are given mainly through

$$
p+p \quad \longrightarrow \pi^{+}+\pi^{-}+\pi^{0}+X
$$


Taking into account that neutral pions decay in two gammas, $\pi^{0} \rightarrow \gamma \gamma$, and the minimum energy of photo-pion, $E_{\pi^{0} \text {,min }}$, at rest frame is $m_{\pi^{0}}=139.57 \mathrm{MeV}$, then the minimum observed energy is

$$
\epsilon_{\gamma, \pi^{0}, \min }^{o b s} \simeq \frac{\delta_{D}}{(1+z)} \frac{m_{\pi^{0}}}{2}
$$

Also charged pions decay in neutrinos as follows

$$
\begin{aligned}
& \pi^{+} \rightarrow \mu^{+}+\nu_{\mu} \rightarrow e^{+}+\nu_{e}+\bar{\nu}_{\mu}+\nu_{\mu}, \\
& \pi^{-} \rightarrow \mu^{-}+\bar{\nu}_{\mu} \rightarrow e^{-}+\bar{\nu}_{e}+\nu_{\mu}+\bar{\nu}_{\mu},
\end{aligned}
$$

hence neutrino flux is expected to be accompanied by a $\gamma$-ray flux.

Assuming that accelerated protons interact with thermal particles whose number density lies in the range $10^{-10} \mathrm{~cm}^{-3} \leq n_{p} \leq 10^{-4} \mathrm{~cm}^{-3}$ (Hardcastle et al. 2009; O'Sullivan et al. 2013; Wykes et al. 2013; Stawarz et al. 2013), then the $\gamma$-ray spectrum, $\left(d N_{\gamma} / d \epsilon_{\gamma}\right)_{\pi^{0}}$, produced by pp interactions is (Atoyan \& Dermer 2003; Fraija et al. 2012; Aharonian 2002; Hardcastle et al. 2009)

$$
f_{\pi^{0}, p p}\left(E_{p}\right) E_{p}\left(\frac{d N_{p}}{d E_{p}}\right)^{o b s} d E_{p}=\epsilon_{\gamma, \pi^{0}}\left(\frac{d N_{\gamma}}{d \epsilon_{\gamma}}\right)_{\pi^{0}}^{o b s} d \epsilon_{\gamma, \pi^{0}}
$$

here $f_{\pi^{0}, p p} \approx t_{l o b e} / t_{p p}=t_{\text {lobe }} n_{p} k_{p p} \sigma_{p p}$ is the fractional power released, $\sigma_{p p} \simeq 30(0.95+$ $0.06 \ln (\mathrm{E} / \mathrm{GeV}) \mathrm{mb}$ is the nuclear interaction cross section, $k_{p p}=1 / 2$ is the inelasticity coefficient, $n_{p}$ is the thermal particle density, $t_{l o b e}$ is the age of the lobe, and $t_{p p}$ is the characteristic cooling time for this process. Taking into account that a pion carries $33 \%$ of the proton energy $(\xi=0.33)$ and supposing that $\gamma$-ray spectrum at $\mathrm{GeV}$ energy range is produced by a simple proton power law,

$$
\left(\frac{d N_{p}}{d E_{p}}\right)^{o b s}=A_{p}\left(\frac{E_{p}^{o b s}}{\mathrm{GeV}}\right)^{-\alpha}
$$

where $A_{p}$ is the proportionality constant normalized to $\mathrm{GeV}$ and $\alpha$ is the spectral index, then the observed $\gamma$-ray spectrum is

$$
\left(\epsilon_{\gamma}^{2} \frac{d N_{\gamma}}{d \epsilon_{\gamma}}\right)_{\pi^{0}}^{o b s}=A_{p p, \gamma}\left(\frac{\epsilon_{\gamma, \pi^{0}}^{o b s}}{\mathrm{GeV}}\right)^{2-\alpha}
$$


where

$$
A_{p p, \gamma}=f_{\pi^{0}, p p}(2 / \xi)^{2-\alpha} A_{p} \mathrm{GeV}^{2}
$$

and the proton luminosity, $L_{p} \simeq 4 \pi F_{p}=4 \pi \int E_{p} \frac{d N_{p}}{d E_{p}} d E_{p}$, can be written as

$$
L_{p}=\frac{4 \pi(\xi / 2)^{2-\alpha}}{(\alpha-2)} D_{z}^{2} f_{\pi^{0}, p p}^{-1} A_{p p, \gamma}\left(\frac{E_{p, \min }}{\mathrm{GeV}}\right)^{2-\alpha}
$$

here $E_{p, \min }$ corresponds to the proton energy at GeV energies. Eq. 13 shows the contribution of pp interactions to the $\gamma$-ray spectrum for each of the lobes.

\section{Production of UHE cosmic rays}

It has been proposed that astrophysical sources accelerating UHECRs could produce HE $\gamma$-rays and neutrinos by proton interactions with photons at the source and/or the surrounding radiation and matter. We propose that the spectrum of accelerated protons is extended from $\mathrm{GeV}$ to $\sim 10^{20} \mathrm{eV}$ energies and can be also determined through signature ( $\gamma$-ray flux) produced at $\mathrm{GeV}$ energies. This $\gamma$-ray flux is correlated with proton flux through eq. 14, In addition, we correlate the $\gamma$-ray and neutrino fluxes to find the parameters of neutrino spectrum (Becker 2008). Based on these correlations, we are going to calculate the number of events for these spectra at energy ranges of PAO and IceCube.

\subsection{UHE protons}

PAO studying the composition of the high-energy showers found that the distribution of their properties was situated in somewhere between pure p and pure Fe at $57 \mathrm{EeV}$ (Yamamoto 2008; Pierre Auger Collaboration \& et al. 2008; Unger et al. 2007). By contrast, HiRes data are consistent with a dominant proton composition at these energies, but uncertainties in the shower

properties (Unger et al. 2007) and in the particle physics extrapolated to this extreme energy scale 
(Engel 2008) preclude definitive statements about the composition. At least two events of the UHECRs observed by PAO were detected (Pierre Auger Collaboration \& et al. 2007, 2008) inside a $3.1^{\circ}$ circle centered at Cen A.

\subsubsection{Mechanisms of UHECR acceleration}

The maximum energy required for acceleration of UHECRs is limited by both the size $(R)$ and magnetic field $(B)$ of the emission region, $E_{\max }=Z e B R \Gamma$ (Hillas 1984). Additional limitations are mainly due to radiative losses or available time when particles diffuse through the magnetized region. In Cen A, a short distance $\left(\sim 10^{15} \mathrm{~cm}\right)$ from the black hole $(\mathrm{BH})$, the emission region is limited by the variability time scale $R=r_{d}=\frac{c \delta_{D}}{(1+z)^{2}} d t^{o b s}$, hence the maximum energy required is (Abdo \& et al. 2010b; Sahu et al. 2012)

$$
E_{\text {max }}=4 \times 10^{19} \mathrm{eV} B_{0.8} d t_{5}^{o b s} \Gamma_{0.85}
$$

A hundred of $\mathrm{kpc}$ distance from the $\mathrm{BH}$, particles are accelerated inside the lobes, therefore the emission region is limited by the size of the lobes, then the maximum energy is

$$
E_{\text {max }}=Z e B R \Gamma
$$

with $\mathrm{R}=100 \mathrm{kpc}$ corresponding to a volume of $V=1.23 \times 10^{71} \mathrm{~cm}^{-3}$ and $B$ the magnetic field of lobes. The acceleration and diffuse time scales are

$$
t_{a c c} \simeq 2 \pi \frac{E_{\max }}{e B}
$$

and

$$
t_{\text {diff }} \simeq \frac{3}{2 \pi} \frac{R^{2} e B}{E_{\max }}
$$

respectively. As the lobes are inflated by jets in the surrounding medium, accelerated protons are injected inside by the jet, and confined within the lobes, by means of resonant Fermi-type 
processes (Hardcastle et al. 2009). The non-thermal and the upper limit thermal pressure in the lobes are $p_{n t h} \simeq\left(U_{e}+U_{B}+U_{p}\right)$ and $\mathrm{p}_{t h}=n_{p} K T$, respectively, where $U_{p} \sim 2 L_{p} t_{l o b e} / V$ is the energy density of accelerated proton, $\mathrm{k}$ is the Boltzmann constant and $\mathrm{T}$ is the temperature. Assuming an equipartition between magnetic field and relativistic electron, $U_{e}=\lambda_{e, B} U_{B}$, then the non-thermal pressure and the total energy can written as

$$
p_{n t h} \simeq U_{B}\left(1+\lambda_{e, B}\right)+2 L_{p} \frac{t_{l o b e}}{V}
$$

and

$$
E_{\text {tot }} \simeq U_{B} V\left(1+\lambda_{e, B}\right)+2 L_{p} t_{l o b e}
$$

respectively. As one can observe from the values of the emission region in the jet (eq. 16), protons might be or not accelerated up to energies above $40 \mathrm{EeV}$, depending on the variability time scale and strength of the magnetic field. Hence, it is important to mention that protons could have a hybrid acceleration mechanism, partially in the jet and finally in the lobes.

On the other hand, supposing that the $\mathrm{BH}$ jet has the power to accelerate particles up to ultra-high energies through Fermi processes, then from the equipartition magnetic field $\epsilon_{B}$ and during flaring intervals for which the apparent isotropic luminosity can reach $\approx 10^{45} \mathrm{erg} \mathrm{s}^{-1}$, one can derive the maximum particle energy of accelerated UHECRs as (Dermer et al.|2009; Fraija et al. 2012)

$$
E_{\text {max }} \approx 1.0 \times 10^{20} \frac{Z e}{\phi} \frac{\sqrt{\epsilon_{B} L / 10^{45} \mathrm{erg} \mathrm{s}^{-1}}}{\beta^{3 / 2} \Gamma} \mathrm{eV},
$$

where $\Gamma=1 / \sqrt{1-\beta^{2}}, \phi \simeq 1$ is the acceleration efficiency factor and $\mathrm{Z}$ is the atomic number. Other more exotic mechanisms that have been described in the literature are magnetic reconnection and stochastically acceleration by temperatures. In the magnetic reconnection framework, the free energy stored in the helical configuration can be converted to particle kinetic energy in the region where the un-reconnected (upstream) magnetized fluid converges into the reconnection layer, resulting in a continuously charged particle acceleration. Some authors (Birk \& Lesch 2000; Giannios 2010; Wykes et al. 2013) have proposed that this mechanism might accelerate UHECRs 
either in the jet or in the giant lobes. Recently, Wykes et al. (2013) proposed that UHECRs could be stochastically accelerated by high temperatures, being responsible for the self-consistency between the entrainment calculations and the missing pressure in the lobes.

\subsubsection{The expected Number of UHECR}

To determine the number of UHECRs, we take into account the PAO exposure, which for a point source is given by $\Xi t_{o p} \omega\left(\delta_{s}\right) / \Omega_{60}$, where $\Xi t_{o p}=\left(\frac{15}{4}\right) 9 \times 10^{3} \mathrm{~km}^{2} \mathrm{yr}, t_{o p}$ is the total operational time (from 1 January 2004 until 31 August 2007), $\omega\left(\delta_{s}\right) \simeq 0.64$ is an exposure correction factor for the declination of Cen $\mathrm{A}$, and $\Omega_{60} \simeq \pi$ is the Auger acceptance solid angle (Cuoco \& Hannestad 2008). The expected number of UHECRs for each of the lobes of Cen A observed above an energy of $60 \mathrm{EeV}$ is given by

$$
N_{U H E C R}=(\text { PAO Expos. }) \times N_{p},
$$

where $N_{p}$ is calculated from a simple and broken power law of the proton spectrum at energies higher than $60 \mathrm{EeV}$. In the first case, considering a simple power law eq. (12) and from eqs. (14) and (23), the expected number of UHECRs is

$$
N_{U H E C R}=\frac{\Xi t_{o p} \omega\left(\delta_{s}\right)(\xi / 2)^{2-\alpha}}{\Omega_{60}(\alpha-1)} f_{\pi^{0}, p p}^{-1} A_{p p, \gamma}\left(\frac{60 E e V}{\mathrm{GeV}}\right)^{-\alpha+2} \mathrm{EeV}^{-1}
$$

that corresponds to an isotropic UHECR luminosity (Dermer et al. 2009)

$$
L_{U H E C R}=\frac{4 \pi(\xi / 2)^{2-\alpha}}{(\alpha-2)} D_{z}^{2} f_{\pi^{0}, p p}^{-1} A_{p p, \gamma}\left(\frac{60 \mathrm{EeV}}{\mathrm{GeV}}\right)^{2-\alpha}
$$

In the second case, we assume that the proton spectrum is not extended continually up to $\sim 10^{20}$, but broken at some energy less than $60 \mathrm{EeV}$. Hence, it can be written as

$$
\frac{d N_{p}}{d E_{p}}=A_{p} \begin{cases}\left(\frac{E_{p}}{\mathrm{GeV}}\right)^{-\alpha} & E_{p}<E_{p, b} \\ \left(\frac{E_{p, b}}{\mathrm{GeV}}\right)^{-\alpha+\beta}\left(\frac{E_{p}}{\mathrm{GeV}}\right)^{-\beta} & E_{p, b} \leq E_{p}\end{cases}
$$


where $\beta$ and $E_{p, b}$ are the higher spectral index and break proton energy, respectively. In this case, the number of expected events is

$$
N_{U H E C R}=\frac{\Xi t_{o p} \omega\left(\delta_{s}\right)(\xi / 2)^{2-\alpha}}{\Omega_{60}(\alpha-1)} f_{p p}^{-1} A_{p p, \gamma}\left(\frac{E_{p, b}}{\mathrm{GeV}}\right)^{-\alpha+\beta}\left(\frac{60 \mathrm{EeV}}{\mathrm{GeV}}\right)^{-\beta+2} \mathrm{EeV}^{-1}
$$

where in eq. (27), $\beta$ and $E_{p, b}$ are the unknown quantities.

\subsection{HE Neutrinos}

Neutrinos are detected when they interact inside the instrumented volume. The path length $\mathrm{L}(\theta)$ traversed within the detector volume by a neutrino with zenith angle $\theta$ is determined by the detector's geometry. At a first approximation, neutrinos are detected if they interact within the detector volume, i.e. within the instrumented distance $\mathrm{L}(\theta)$. The probability of interaction for a neutrino with energy $E_{\nu}$ is

$$
P\left(E_{\nu}\right)=1-\exp \left[-\frac{L}{\lambda_{\nu}\left(E_{E_{\nu}}\right)}\right] \cong \frac{L}{\lambda_{\nu}\left(E_{\nu}\right)}
$$

where the mean free path in ice is

$$
\lambda_{\nu}\left(E_{\nu}\right)=\frac{1}{\rho_{i c e} N_{A} \sigma_{\nu N}\left(E_{\nu}\right)} .
$$

Here $\rho_{i c e}=0.9 \mathrm{~g} \mathrm{~cm}^{-3}$ is the density of the ice, $\mathrm{N}_{A}=6.022 \times 10^{23} \mathrm{~g}^{-1}$ and $\sigma_{\nu N}$ is the neutrinonucleon cross section. A neutrino flux, $\mathrm{dN}_{\nu} / \mathrm{d} E_{\nu}$, crossing a detector with energy threshold $E_{\nu}^{t h}$ and cross-sectional area $A\left(E_{\nu}, \theta\right)$ facing the incident beam will produce

$$
N_{e v}=T \int_{E_{\nu}^{t h}} A\left(E_{\nu}\right) P\left(E_{\nu}\right) \frac{d N_{\nu}}{d E_{\nu}} d E_{\nu}
$$

events after a time $T$. Furthermore, the "effective" detector area $\mathrm{A}\left(E_{\nu}, \theta\right)$ is clearly also a function of zenith angle $\theta$. In practice, $\mathrm{A}\left(E_{\nu}, \theta\right)$ is determined as a function of the incident neutrino direction and zenith angle by a full-detector simulation including the trigger. It is of the order of 1 
$\mathrm{km}^{2}$ for IceCube, so the effective volume for showers is $\mathrm{V}_{\text {eff }} \approx \mathrm{A}\left(E_{\nu}, \theta\right) \mathrm{L}(\theta) \approx 2 \mathrm{~km}^{3}$. Finally the expected event rate is

$$
N_{e v} \approx T \rho_{i c e} N_{A} V_{e f f} \int_{E_{t h}}^{\infty} \sigma_{\nu N}\left(E_{\nu}\right) \frac{d N_{\nu}}{d E_{\nu}} d E_{\nu}
$$

where $\mathrm{E}_{t h}$ is the threshold energy, $\sigma_{\nu N}\left(E_{\nu}\right)=5.53 \times 10^{-36}\left(E_{\nu} / G e V\right)^{0.363} \mathrm{~cm}^{2}$ is the charged current cross section (Gandhi et al. 1998).

Proposing that the neutrino spectrum can be written as

$$
\frac{d N_{\nu}}{d E_{\nu}}=A_{\nu}\left(\frac{E_{\nu}}{\mathrm{GeV}}\right)^{-\alpha_{\nu}},
$$

where the normalization factor, $\mathrm{A}_{\nu}$, is calculated by correlating the neutrino flux luminosity with the $\mathrm{GeV}$ photon flux (Becker 2008). This correlation is given by

$$
\int \frac{d N_{\nu}}{d E_{\nu}} E_{\nu} d E_{\nu}=\int \frac{d N_{\gamma}}{d E_{\gamma}} E_{\gamma} d E_{\gamma} .
$$

Here, we have used $K=1$ for $p p$ interactions (see, e.g. Halzen 2007, and reference therein). Assuming that the spectral indices for neutrino and $\gamma$-ray spectrum are similar $\alpha \simeq \alpha_{\nu}$ (Becker 2008), and taking into account that each neutrino carries $5 \%$ of the proton energies $\left(E_{\nu}=1 / 20 E_{p}\right)$ and each photon carries $16.7 \%$ of proton energy (Halzen 2013), then the normalization factors are related by

$$
A_{\nu}=A_{p p, \gamma}(10 \xi)^{-\alpha+2} \mathrm{GeV}^{-2},
$$

where $\mathrm{A}_{p p, \gamma}$ is given by Eq. (14).

If we assume that the neutrino spectrum extends continually over the whole energy range (Cuoco \& Hannestad 2008), then the expected number of neutrinos is

$$
N_{e v} \approx \frac{T \rho_{i c e} N_{A} V_{e f f}}{\alpha-1.363} A_{\nu}\left(5.53 \times 10^{-36} \mathrm{~cm}^{2}\right)\left(\frac{E_{\nu, t h}}{\mathrm{GeV}}\right)^{\alpha+1.363} \mathrm{GeV}^{-1} .
$$

and if it is broken at energy $E_{\nu, b}=\frac{1}{20} E_{p, b}$, then

$$
\frac{d N_{\nu}}{d E_{\nu}}=A_{\nu} \begin{cases}\left(\frac{E_{\nu}}{\mathrm{GeV}}\right)^{-\alpha} & E_{\nu}<E_{\nu, b}, \\ \left(\frac{E_{\nu, b}}{\mathrm{GeV}}\right)^{-\alpha+\beta}\left(\frac{E_{\nu}}{\mathrm{GeV}}\right)^{-\beta} & E_{\nu, b} \leq E_{\nu} .\end{cases}
$$


For this case, the expected event is

$$
\begin{aligned}
N_{e v} \approx & \frac{T \rho_{i c e} N_{A} V_{e f f}}{\alpha-1.363} A_{\nu}\left(5.53 \times 10^{-36} \mathrm{~cm}^{2}\right) \\
& \times\left[\left(\frac{E_{\nu, t h}}{\mathrm{GeV}}\right)^{-\alpha+1.363}+\frac{\alpha-\beta}{\beta-1.363}\left(\frac{E_{\nu, b}}{\mathrm{GeV}}\right)^{-\alpha+1.363}\right] \mathrm{GeV}^{-1} .
\end{aligned}
$$

where the higher spectral index, $\beta$, is given by the broken power law of proton spectrum.

\section{Analysis and Results}

We have developed a synchrotron emission and pp interaction model to describe the spectra for the north and south lobes of Cen A. In the synchrotron radiation model we have used an electron distribution described by a broken power law (eq. 1) with the minimum Lorentz factor calculated through electron density and electron energy density (eq. 2). The synchrotron spectrum obtained (eq. 5) depends on magnetic and electron energy densities, electron density, size of emission region and cooling time scale characteristic for this process, through the synchrotron normalization constant (eq. 6) and break energies (characteristic and cut off) (eq. 3). Taking into account that the jet extends out to $\sim 3 \mathrm{kpc}$ in projection in radio (Hardcastle et al. 2007, 2003; Tingay et al. 1998), we consider an emission region scale of this size. Also we have supposed that the magnetic and electron energy densities are equipartitioned through the parameter $\lambda_{e, B}=U_{e} / U_{B}$. In the pp interaction model, we have considered accelerated protons described by a simple power law (eq. 12) which could be accelerated in the jet or/and the size of the lobe and furthermore interact with thermal particles in the lobes. The spectrum generated by this process (eq. 13) depends on the proton luminosity (through $A_{p}$ ), number density of thermal particles, age of the lobe and spectral index. The age of the lobe can be estimated through cooling time scale of radiating electrons (Hardcastle et al. 2009) and the number density lies in the range $10^{-10} \mathrm{~cm}^{-3} \leq n_{p} \leq 10^{-4} \mathrm{~cm}^{-3}$.

To find the best fit of the set of model parameters with data for each lobe, we use the method 
of Chi-square $\left(\chi^{2}\right)$ minimization (Brun \& Rademakers 1997). We have fitted WMAP and Fermi data for each of the lobes with synchrotron emission (eqs. 3] and 5) and pp interaction (eq. 13), respectively. As shown in appendix A, firstly we found the photon spectral index $(\alpha)$ and the normalization constant $\left(A_{p p, \gamma}\right)$ of the $\gamma$-ray spectrum generated by pp interaction model. Secondly, with the fitting spectral index we fit WMAP data with the synchrotron model to find the break energies (characteristic $\epsilon_{\gamma, m}^{o b s}$ and cut off $\epsilon_{\gamma, c}^{o b s}$ ) and the normalization constant characteristic of this process $\left(A_{s y n, \gamma}\right)$. Finally, after fitting the SED of each lobe, we plot fig. 1 presenting also the best set of these parameters: pp interaction parameters in table A1 and synchrotron radiation parameters in table A2 (see appendix A).

As shown in table 1 , it can be seen that the values of normalization constant $A_{p p, \gamma}$ and photon spectral index $(\alpha)$ of the $\gamma$-ray spectra for each of the lobes are $[5.10 \pm 0.96] \mathrm{erg} \mathrm{cm}^{-2} \mathrm{~s}^{-1}$ (north) and $[8.07 \pm 1.58] \mathrm{erg} \mathrm{cm}^{-2} \mathrm{~s}^{-1}$ (south), and 2.519 (north) and 2.598 (south), respectively, which were firstly obtained by Abdo \& et al. (2010a). From the values of the best set of parameters obtained with the synchrotron model (table A2 and eqs. 6 and 3), we plot the synchrotron cooling time scale $\left(t_{\text {syn }}\right)$, the electron density $\left(N_{e}\right)$ and equipartition parameter $\left(\lambda_{e, B}\right)$ as a function of magnetic field $(B)$, as shown in fig. 2. For the north lobe, considering the value of equipartition parameter $\lambda_{e, B}=4.3$ (Abdo \& et al. 2010a), we found the value of magnetic field $\mathrm{B}=3.41 \mu \mathrm{G}$, and then the values of synchrotron cooling time $t_{\text {syn }}=55.1 \mathrm{Myr}$ and electron density $N_{e}=2.1 \times 10^{10} \mathrm{~cm}^{-3}$. For the south lobe, considering the value of equipartition parameter $\lambda_{e, B}=1.8$ (Abdo \& et al. 2010a), we found the value of magnetic field $\mathrm{B}=6.19 \mu \mathrm{G}$, and then the values of synchrotron cooling time $t_{s y n}=27 \mathrm{Myr}$ and electron density $N_{e}=3.9 \times 10^{10} \mathrm{~cm}^{-3}$. On the other hand, from the values of the observed quantities and parameters given in tables A1 and A2, firstly we analyze the contributions of $\mathrm{p} \gamma$ interactions and inverse Compton scattering of $\mathrm{CMB}$ and EBL to the $\gamma$-ray spectra and secondly, from pp interactions we correlate the $\gamma$-ray, UHECRs and neutrino fluxes to estimate the number of UHE protons and neutrinos expected in PAO and IceCube, respectively. These estimations are done, assuming that these spectra are 
extended up to the energy range of each of the experiments.

Relativistic electrons may upscatter synchrotron photons up to higher energies given by

$$
E_{\gamma, k}^{i c} \simeq \gamma_{e, m / c}^{2} E_{\gamma, k}
$$

where the index $k$ represents the CMB and EBL photons (Hauser \& Dwek 2001;

Georganopoulos et al. 2008; Raue \& Mazin 2008; Dermer 2013). Taking into account the radiation power typical of this process $d E / d t=4 / 3 \sigma_{T} \beta^{2} \gamma_{e}^{2} U_{\gamma}$ and performing a process similar to that done with synchrotron emission, the IC spectrum can be written as

$$
\epsilon_{\gamma}^{i c^{2}} N_{\gamma}\left(\epsilon_{\gamma}^{i c}\right)=A_{i c, \gamma} \begin{cases}\left(\frac{\epsilon_{\gamma}^{i c}}{\epsilon_{\gamma, m}^{c}}\right)^{4 / 3} & \epsilon_{\gamma}^{i c, o b s}<\epsilon_{\gamma, m}^{i c, o b s}, \\ \left(\frac{\epsilon_{\gamma}^{i c}}{\epsilon_{\gamma, m}^{i c}}\right)^{-(\alpha-3) / 2} & \epsilon_{\gamma, m}^{i c, o b s}<\epsilon_{\gamma}^{i c, o b s}<\epsilon_{\gamma, c}^{i c, o b s}, \\ \left(\frac{\epsilon_{\gamma, c}^{i c}}{\epsilon_{\gamma, m}^{c}}\right)^{-(\alpha-3) / 2}\left(\frac{\epsilon_{\gamma}^{i c}}{\epsilon_{\gamma, c}^{i c}}\right)^{-(\alpha-2) / 2}, & \epsilon_{\gamma, c}^{i c, o b s}<\epsilon_{\gamma}^{i c, o b s}<\epsilon_{\gamma, \text { max }}^{i c, o b s}\end{cases}
$$

where

$$
A_{i c, \gamma}=\simeq \frac{\sqrt{8 \pi} \sigma_{T} \beta^{2}}{9 q_{e} D_{z}^{2}}(1+z)^{-1} \delta_{D} U_{B}^{-1 / 2} N_{e} r_{d}^{3} U_{\gamma} E_{\gamma, k},
$$

and $U_{\gamma}$ is the photon energy density of CMB and EBL. Replacing the electron Lorentz factors and the typical photon energies of CBM and EBL (Dermer 2013) (eqs. (3) and (2)) in eqs. (38), (39) and (40) and from the best set of parameters, we plot these contributions, as shown in fig. 3. In this figure, we can notice that a superposition of inverse Compton of CMB and EBL, and pp interactions could describe satisfactorily the observed Fermi-LAT fluxes. Otherwise, $\mathrm{p} \gamma$ interactions take place when accelerated protons collide with target photons. The single-pion production channels are $p+\gamma \rightarrow n+\pi^{+}$and $p+\gamma \rightarrow p+\pi^{0}$, where the relevant pion decay chains are $\pi^{0} \rightarrow 2 \gamma, \pi^{+} \rightarrow \mu^{+}+\nu_{\mu} \rightarrow e^{+}+\nu_{e}+\bar{\nu}_{\mu}+\nu_{\mu}$ and $\pi^{-} \rightarrow \mu^{-}+\bar{\nu}_{\mu} \rightarrow e^{-}+\bar{\nu}_{e}+\nu_{\mu}+\bar{\nu}_{\mu}$. Taking into account that $\pi^{0}$ carries $20 \%$ of the proton's energy and that each produced photon shares the same energy then, the observed HE photon is given by

$$
E_{\gamma, H E}^{o b s} \simeq 0.5 \frac{\delta_{D}^{2}\left(m_{\Delta}^{2}-m_{p}^{2}\right)}{(1+z)^{2}}\left(E_{\gamma, L E}^{o b s}\right)^{-1},
$$

where $E_{\gamma, L E}^{o b s}$ corresponds to low-energy (LE) photons. Based on eq. (41), it is necessary that target photons should be in the energy range of $E_{\gamma, L E} \sim(30-460) \mathrm{MeV}$ for a full description of 
$\gamma$-ray spectra. Although a more robust analysis should be done such as a calculation of density of target photons, optical depth, rate of energy loss, etc, a simple calculation shows that this process needs seed photons with energies from tens to hundreds of $\mathrm{MeV}$ which is completely different to $\gamma$-ray spectra observed by LAT. Hence, there is no contribution of $\mathrm{p} \gamma$ emission to the $\gamma$-ray spectra.

On the other hand, in addition to the analysis performed and showed here about the SED of the lobes, we are going to see whether there is any correlation of $\gamma$-ray spectra and UHECRs collected with PAO. Estimating the age of the lobes by means of synchrotron cooling time, $t_{\text {lobe }} \simeq t_{\text {syn }}$ (Hardcastle et al. 2009) and the acceleration (eq. 18) and diffuse (eq. 18) time scales with the magnetic field found we obtain that the acceleration time, diffuse time and the age of the lobes are 0.65 (0.37) Myr, 0.49 (0.89) Myr and 55.1 (27) Myr for the north (south) lobe, respectively. Comparing the time scales, $t_{\text {acc }} \sim t_{\text {diff }} \ll t_{\text {lobe }}$, one can calculate that the maximum proton energies required are $E_{p, \max }=8.67(15.81) \times 10^{19} \mathrm{eV}$ for the north (south) lobes, hence we demonstrate that protons could be accelerated up to energies as high as $10^{20} \mathrm{eV}$ in the lobes. Following our analysis, we replace the fitting parameters $\mathrm{t}_{l o b e}, \alpha$ and $A_{p p, \gamma}$ in eqs. 27 and 25 to calculate the proton luminosity from $\sim \mathrm{GeV}$ to $10^{11} \mathrm{GeV}$ and also estimate the number of UHE protons expected with PAO. For this calculation we take into account the thermal number density in the range $10^{-10} \mathrm{~cm}^{-3} \leq n_{p} \leq 10^{-4} \mathrm{~cm}^{-3}$. As shown in fig. 4, one can see that proton luminosity increases as thermal density decreases and it decreases as energy increases. In this plot there are two interesting ranges, the $\mathrm{GeV}$ and $\mathrm{EeV}$ ranges. In $\mathrm{GeV}$ range which is connected directly with the Fermi fluxes, the proton luminosities in the north(south) lobe at some GeV energy are $3.7(7.7) \times 10^{43} \mathrm{erg} \mathrm{s}^{-1}$ and $3.7(7.7) \times 10^{49} \mathrm{erg} \mathrm{s}^{-1}$ for minimum $\left(n_{p}=10^{-4} \mathrm{~cm}^{-3}\right)$ and maximum $\left(n_{p}=10^{-10} \mathrm{~cm}^{-3}\right)$ thermal particle densities considered and in the EeV range the luminosities are $3.11(2.31) \times 10^{38} \mathrm{erg} \mathrm{s}^{-1}$ and $3.11(2.31) \times 10^{44} \mathrm{erg} \mathrm{s}^{-1}$ for the same thermal particle densities. The values of luminosity at GeV energy range are of the same order as those found by Hardcastle et al. (2009); Wykes et al. (2013); Abdo \& et al. (2010a) and at EeV energy 
range it is also in accordance with Hardcastle et al. (2009); Dermer et al. (2009). In fig. 5, the number of events as a function of thermal density is plotted when a power law was considered. As shown, the expected number decreases as thermal particle density decreases, reaching a minimum value of 3.52 (north lobe) and 3.0 (south lobe) with density is equal to $10^{-4} \mathrm{~cm}^{3}$, see table 1 .

\begin{tabular}{ccc}
\hline \hline & North Lobe & South Lobe \\
\hline$n_{p}\left(\mathrm{~cm}^{-3}\right)$ & Number of UHECRs & Number of UHECRs \\
\hline $10^{-4}$ & 3.52 & 3.00 \\
$10^{-5}$ & 35.2 & $30 \times 10^{-2}$ \\
$10^{-6}$ & 352 & 300 \\
$10^{-7}$ & $3.52 \times 10^{3}$ & $3.00 \times 10^{3}$ \\
$10^{-8}$ & $3.52 \times 10^{4}$ & $3.00 \times 10^{4}$ \\
$10^{-9}$ & $3.52 \times 10^{5}$ & $3.00 \times 10^{5}$ \\
$10^{-10}$ & $3.52 \times 10^{6}$ & $3.00 \times 10^{6}$ \\
\hline
\end{tabular}

Table 1. Number of expected events (UHECRs) in PAO as a function of number density of thermal particles from the north and south lobe of Cen A.

Considering a simple power law and the number density of thermal particles equal to $10^{-4} \mathrm{~cm}^{3}$, the result of number of expected UHECRs is very similar to that reported by PAO. Also taking into account this number density and from eqs. 20 and 21 we estimate the pressures and energies in the lobes, see table 2 .

\begin{tabular}{|c|c|c|c|}
\hline & & North Lobe & South Lobe \\
\hline & Symbol & Values & Values \\
\hline Non-thermal pressure $\left(\mathrm{dyn} \mathrm{cm}^{-2}\right)$ & $\mathrm{P}_{n t h}$ & $3.5 \times 10^{-12}$ & $5.4 \times 10^{-12}$ \\
\hline Thermal pressure $\left(\mathrm{dyn} \mathrm{cm}^{-2}\right)$ & $P_{t h}$ & $0.9 \times 10^{-13}$ & $0.9 \times 10^{-13}$ \\
\hline Proton density energy $\left(\mathrm{erg} \mathrm{cm}^{-3}\right.$ ) & $U_{p}$ & $1.06 \times 10^{-12}$ & $1.07 \times 10^{-12}$ \\
\hline Total energy (erg) & $E_{t o t}$ & $4.4 \times 10^{59}$ & $6.6 \times 10^{59}$ \\
\hline
\end{tabular}

Table 2. Distributions of pressures and energies in the north and south lobe of Cen A. These values have been obtained for a volume $1.3 \times 10^{71} \mathbf{c m}^{-3}$, number density of thermal particles $n_{p} \sim 10^{-4} \mathrm{~cm}^{3}$ and temperature $\sim 10^{7} \mathrm{~K}$.

As shown in table 2, the bigger contribution of pressure exerted on the lobes comes from non-thermal particles and the contribution of protons to pressure and energy is although not 
dominant if significant.

For this density and eq. (35), we calculate the number of neutrinos expected per year in IceCube for neutrino threshold energy equal to $E_{\nu, t h}=1 \mathrm{TeV}$ (see table 3). In this table, we can see that non neutrinos are expected from the lobes.

\begin{tabular}{ccc}
\hline \hline & North Lobe & South Lobe \\
\hline$E_{\nu, t h}$ & $N_{\text {ev }} / T(\text { year })^{-1}$ & $N_{\text {ev }} / T(\text { year })^{-1}$ \\
\hline $1 \mathrm{TeV}$ & $9.41 \times 10^{-2}$ & $7.47 \times 10^{-2}$ \\
\hline
\end{tabular}

Table 3. Number of neutrinos expected on IceCube from north and south lobes of Cen A. This number is calculated taking into account the number density of thermal particles and neutrino threshold energies equal to $n_{p}=10^{-4} \mathrm{~cm}^{-3}$ and, $E_{\nu, t h}=\mathbf{1} \mathrm{TeV}$, respectively.

From table 1 , one can see that for any number density below $10^{-4} \mathrm{~cm}^{-3}$, the expected UHECRs would be much higher than those reported by PAO, hence these densities should be excluded when a simple power law is considered, but not when we give careful consideration to a broken proton spectrum. In other words, taking into account a number density of less than $10^{-4} \mathrm{~cm}^{-3}$, we could expect more or less events only when a broken proton spectrum is considered (eqs. 26 and 27). In fig 6, contour plots of the broken spectrum parameters are plotted, higher spectral index $(\beta)$ and break energy $\left(E_{p, b}\right)$ as a function of number density for which PAO would expect one and two events from each of the lobes. In these graphs can be seen that $\beta$ is higher in the south lobe, as one event is expected and the number density is smaller. For instance, for the break proton energy $\left(E_{p, b}=2.04 \times 10^{17} \mathrm{eV}\right)$ we expect 2 events when $\beta=3.28(3.29)$ and 1 event when $\beta=3.47(3.48)$ for $n_{p}=10^{-5} \mathrm{~cm}^{-3}$ and 2 events when $\beta=3.83(3.91)$ and 1 event when $\beta=3.99$ (4.11) for $n_{p}=10^{-6} \mathrm{~cm}^{-3}$ from north (south) lobe. From the analysis performed for the proton spectrum described by broken power law one can see that it is more favorable when densities of thermal particles are higher.

Additionally, for this case we calculate the number of expected neutrinos in IceCube. From eq. (37), we plot the number of neutrinos as a function of time for a broken power law as shown in figs. 7 and 8 . Taking into account the parameters $\left(\beta\right.$ and $\left.E_{\nu, b}\right)$ for one and two events from the 
north and south lobe, the events per year are reported in tables B1 and B2. As shown in fig. 7 and 8 , as $\beta$ and $E_{\nu, b}$ increase broken power laws become closer up to be overlapped. Assuming a threshold energy of $E_{t h, \nu}=1 \mathrm{TeV}$, it can be seen that less than 0.1 neutrinos are expected in IceCube.

\section{Summary and conclusions}

In the framework of emission processes, we have done an exhaustive analysis to describe the photon spectrum of the lobes of Cen A. In our emission model, firstly we have used synchrotron radiation to fit WMAP data and then estimated the values of magnetic fields, electron number densities as well as the age of the lobes, these values are calculated assuming an equipartition between the magnetic and electron energy density. As shown in fig. 2. these quantities are plotted as a function of magnetic field for a wide range. We estimate the values (see section 4) based on the choices of equipartition parameters; 4.3 and 1.8 for the north and south lobes, respectively (Abdo \& et al. 2010a), therefore the small difference regarding the estimation of the age of north lobe given by (Hardcastle et al. 2009) comes of our election, although the age of south lobe as well as the values of electron number densities in the lobes represent quite accurately Wykes et al. (2013). Secondly, we have fitted Fermi-LAT data with pp interactions in order to obtain the proton luminosity and then the non-thermal and thermal pressure and the total energy in the lobes. Although thermal particle densities in the range $10^{-10} \mathrm{~cm}^{-3} \leq n_{p} \leq 10^{-4} \mathrm{~cm}^{-3}$ describe successfully the $\gamma$-ray spectrum, the density $n_{p} \sim 10^{-4} \mathrm{~cm}^{-3}$ reproduces the value of proton luminosity $\sim 10^{43} \mathrm{erg} \mathrm{s}^{-1}$ which is more realistic in connection with the jet power as well as non-thermal and thermal pressure and total energy which have been estimated by using other methods (O'Sullivan et al. 2013; Stawarz et al. 2013) (see table 2). Again one can see that the small differences come from the election of equipartition parameters and consequently the magnetic field. 
On the other hand, from the values of parameters found we have explored some acceleration mechanisms of UHECRs and showed that protons can be accelerated inside the lobes up to energies as high as $\sim 10^{20} \mathrm{eV}$, then we estimated the number of UHECRs expected in PAO, supposing that proton spectrum extends up to this energy range. We found that few events can be expected on Earth if and only if the thermal particles density is again $\sim 10^{-4} \mathrm{~cm}^{-3}$. However, we investigated the conditions for which few events would arrive taking into account densities $\leq 10^{-5} \mathrm{~cm}^{-3}$. We consider a broken power law for accelerating protons and made contour plots of the spectrum parameters (fig. 6); the higher spectral index $(\beta)$ and the break energy $\left(E_{p, b}\right)$ for which the expected number of UHECRs would be one event for each of the lobes or two events for one lobe.

On the other hand, correlating the $\gamma$-ray and neutrino fluxes we have calculated the number of neutrinos expected in IceCube. Also we have considered a neutrino spectrum described by a simple and broken power law which are extended up to an energy range of IceCube. In both cases the number of neutrinos per year would be less than $0.938 \times 10^{-1}$ and $0.745 \times 10^{-1}$ for the north and south lobes, respectively, which is consistent with the non-detection of HE neutrinos by IceCube in the direction of Cen A (IceCube Collaboration et al. 2013a. b).

On the other hand and as shown in fig. 3, our model is consistent to describe the $\gamma$-ray spectrum with IC-scattering of CMB and EBL firstly proposed and discussed by Abdo \& et al. (2010a); Hardcastle et al. (2009). Additionally, we have briefly introduced $\mathrm{p} \gamma$ interactions and showed that they did not contribute to the $\gamma$-ray spectrum.

We thank the referee for a critical reading of the paper and valuable suggestions. We also thank Charles Dermer, Bin Zhang, Francis Halzen, Ignacio Taboada, William Lee and Antonio Marinelli for useful discussions. NF gratefully acknowledges a Luc Binette-Fundación UNAM Posdoctoral Fellowship. 


\section{REFERENCES}

Abdo, A. A., \& et al. 2010a, Science, 328, 725

-. 2010b, ApJ, 719, 1433

Aharonian, F., \& et al. 2009, ApJ, 695, L40

Aharonian, F. A. 2002, MNRAS, 332, 215

Alvarez, H., Aparici, J., May, J., \& Reich, P. 2000, A\&A, 355, 863

Atoyan, A. M., \& Dermer, C. D. 2003, ApJ, 586, 79

Atwood, W. B., Abdo, A. A., Ackermann, M., et al. 2009, ApJ, 697, 1071

Baity, W. A., \& et al. 1981, ApJ, 244, 429

Becker, J. K. 2008, Phys. Rep., 458, 173

Birk, G. T., \& Lesch, H. 2000, ApJ, 530, L77

Blumenthal, G. R., \& Gould, R. J. 1970, Reviews of Modern Physics, 42, 237

Bowyer, C. S., Lampton, M., Mack, J., \& de Mendonca, F. 1970, ApJ, 161, L1

Brun, R., \& Rademakers, F. 1997, Nuclear Instruments and Methods in Physics Research A, 389, 81

Chiaberge, M., Capetti, A., \& Celotti, A. 2001, MNRAS, 324, L33

Cholis, I., \& Hooper, D. 2012, ArXiv e-prints, arXiv:1211.1974

Crusius, A., \& Schlickeiser, R. 1986, A\&A, 164, L16

Cuoco, A., \& Hannestad, S. 2008, Phys. Rev. D, 78, 023007 
Dermer, C. D. 2013, Sources of GeV Photons and the Fermi Results, ed. F. Aharonian, L. Bergström, \& C. Dermer, 225

Dermer, C. D., \& Menon, G. 2009, High Energy Radiation from Black Holes: Gamma Rays, Cosmic Rays, and Neutrinos

Dermer, C. D., Razzaque, S., Finke, J. D., \& Atoyan, A. 2009, New Journal of Physics, 11, 065016

Engel, R. 2008, in International Cosmic Ray Conference, Vol. 4, International Cosmic Ray Conference, $385-388$

Fraija, N. 2013, MNRAS, arXiv:1310.7061

Fraija, N., González, M. M., Perez, M., \& Marinelli, A. 2012, ApJ, 753, 40

Gandhi, R., Quigg, C., Reno, M. H., \& Sarcevic, I. 1998, Phys. Rev. D, 58, 093009

Georganopoulos, M., Sambruna, R. M., Kazanas, D., et al. 2008, ApJ, 686, L5

Giannios, D. 2010, MNRAS, 408, L46

Gorbunov, D., Tinyakov, P., Tkachev, I., \& Troitsky, S. 2008, Soviet Journal of Experimental and Theoretical Physics Letters, 87, 461

Halzen, F. 2007, Ap\&SS, 309, 407

Halzen, F. 2013, Riv.Nuovo Cim., 036, 81

Halzen, F., \& O’Murchadha, A. 2008, ArXiv e-prints, arXiv:0802.0887

Hardcastle, M. J., Birkinshaw, M., \& Worrall, D. M. 2001, MNRAS, 326, 1499

Hardcastle, M. J., Cheung, C. C., Feain, I. J., \& Stawarz, Ł. 2009, MNRAS, 393, 1041 
Hardcastle, M. J., \& Croston, J. H. 2011, MNRAS, 415, 133

Hardcastle, M. J., Kraft, R. P., \& Worrall, D. M. 2006, MNRAS, 368, L15

Hardcastle, M. J., Worrall, D. M., Kraft, R. P., et al. 2003, ApJ, 593, 169

Hardcastle, M. J., Kraft, R. P., Sivakoff, G. R., et al. 2007, ApJ, 670, L81

Hartman, R. C., Bertsch, D. L., Bloom, S. D., et al. 1999, ApJS, 123, 79

Hauser, M. G., \& Dwek, E. 2001, ARA\&A, 39, 249

Hillas, A. M. 1984, ARA\&A, 22, 425

Hinshaw, G., \& et al. 2009, ApJS, 180, 225

Horiuchi, S., Meier, D. L., Preston, R. A., \& Tingay, S. J. 2006, PASJ, 58, 211

IceCube Collaboration, Aartsen, M. G., Abbasi, R., et al. 2013a, ArXiv e-prints, arXiv:1311.5238

—. 2013b, ArXiv e-prints, arXiv:1304.5356

Israel, F. P. 1998, A\&A Rev., 8, 237

Junkes, N., Haynes, R. F., Harnett, J. I., \& Jauncey, D. L. 1993, A\&A, 269, 29

Kraft, R. P., Forman, W. R., Hardcastle, M. J., et al. 2009, ApJ, 698, 2036

Lenain, J.-P., Boisson, C., Sol, H., \& Katarzyński, K. 2008, A\&A, 478, 111

Liu, R.-Y., \& Wang, X.-Y. 2013, ApJ, 766, 73

Longair, M. S. 1994, High energy astrophysics. Volume 2. Stars, the Galaxy and the interstellar medium.

Mattox, J. R., Bertsch, D. L., Chiang, J., et al. 1996, ApJ, 461, 396 
Moskalenko, I. V., Stawarz, L., Porter, T. A., \& Cheung, C. C. 2009, ApJ, 693, 1261

Murase, K., \& Ioka, K. 2013, Phys. Rev. Lett., 111, 121102

Mushotzky, R. F., Baity, W. A., Wheaton, W. A., \& Peterson, L. E. 1976, ApJ, 206, L45

O’Sullivan, S. P., Feain, I. J., McClure-Griffiths, N. M., et al. 2013, ApJ, 764, 162

Page, L., \& et al. 2003, ApJS, 148, 39

Pierre Auger Collaboration, \& et al. 2007, Science, 318, 938

—. 2008, Astroparticle Physics, 29, 188

Raue, M., \& Mazin, D. 2008, International Journal of Modern Physics D, 17, 1515

Razzaque, S. 2013, ArXiv e-prints, arXiv:1307.7596

Rybicki, G. B., \& Lightman, A. P. 1986, Radiative Processes in Astrophysics

Sahu, S., Zhang, B., \& Fraija, N. 2012, Phys. Rev. D, 85, 043012

Sreekumar, P., Bertsch, D. L., Hartman, R. C., Nolan, P. L., \& Thompson, D. J. 1999, Astroparticle Physics, 11, 221

Stawarz, Ł., Tanaka, Y. T., Madejski, G., et al. 2013, ApJ, 766, 48

Tingay, S. J., Jauncey, D. L., Reynolds, J. E., et al. 1998, AJ, 115, 960

Unger, M., Engel, R., Schüssler, F., Ulrich, R., \& Pierre Auger Collaboration. 2007, Astronomische Nachrichten, 328, 614

Winkler, Jr., P. F., \& White, A. E. 1975, ApJ, 199, L139

Wykes, S., Croston, J. H., Hardcastle, M. J., et al. 2013, A\&A, 558, A19 
Yamamoto, T. 2008, in International Cosmic Ray Conference, Vol. 4, International Cosmic Ray Conference, $335-338$

Yang, R.-z., Sahakyan, N., Wilhelmi, E. D. O., Aharonian, F., \& Rieger, F. 2012, in American Institute of Physics Conference Series, Vol. 1505, American Institute of Physics Conference Series, ed. F. A. Aharonian, W. Hofmann, \& F. M. Rieger, 590-593 

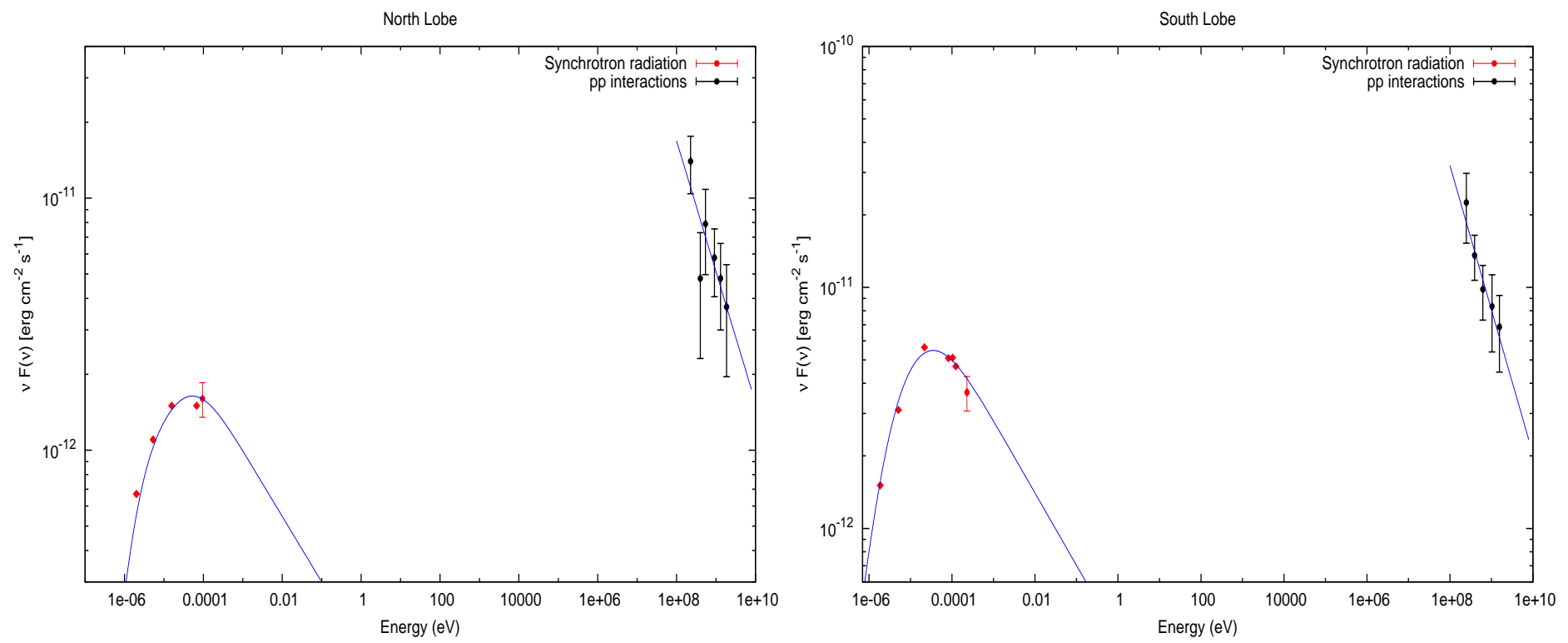

Fig. 1. - Fit of observed SED of the north (left) and south (right) lobes of Cen A. The peak at radio wavelength is described using synchrotron radiation and the $\gamma$-ray spectrum is explained through pp interactions. 

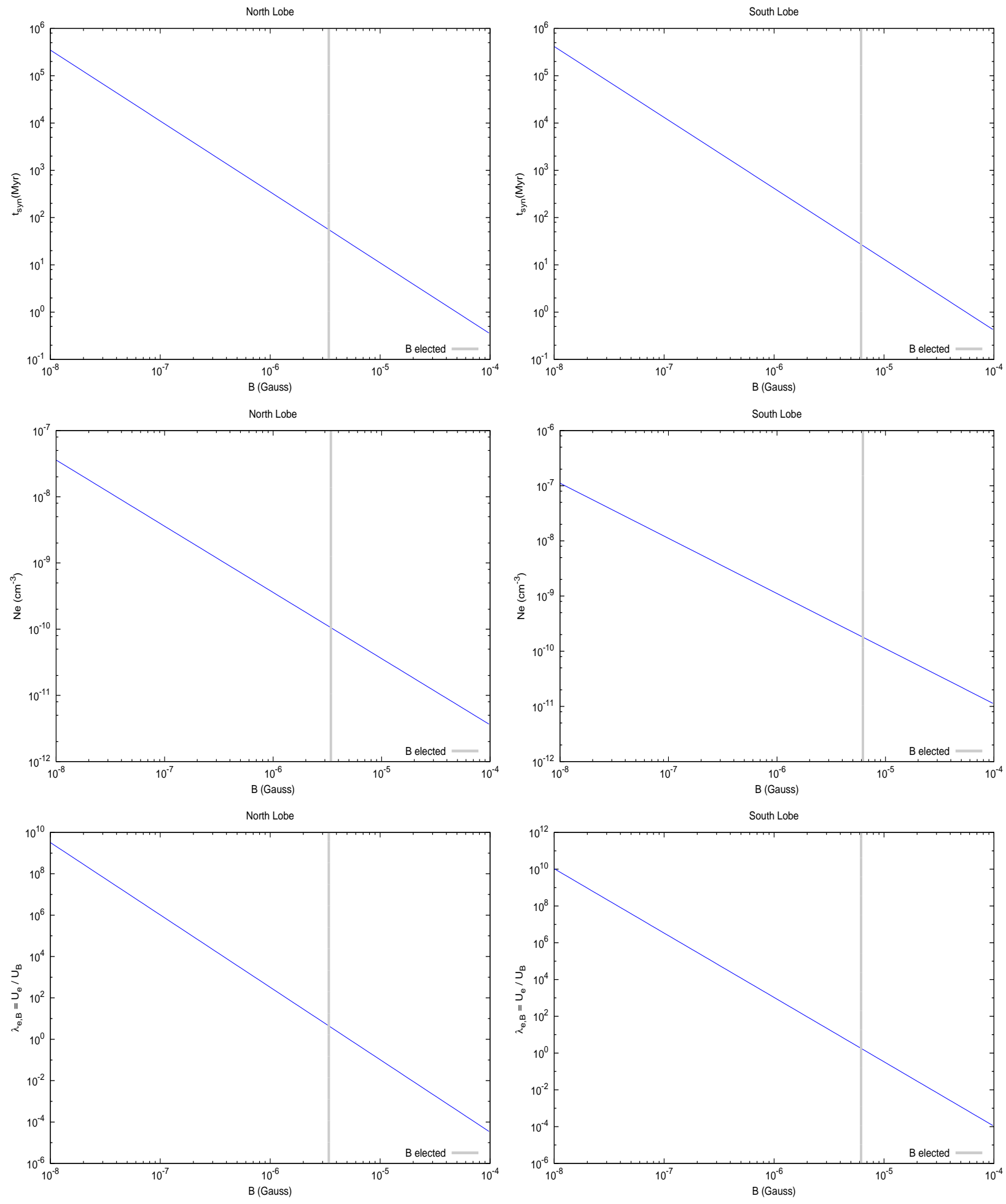

Fig. 2.- Plots of the best set of parameters (synchrotron cooling time $\left(t_{\text {syn }}\right)$, electron density $\left(N_{e}\right)$ and equipartition parameter $\left(\lambda_{e, B}=U_{e} / U_{B}\right)$ as a function of magnetic field (B)) obtained with our synchrotron model for north (left) and south (right) lobes. 

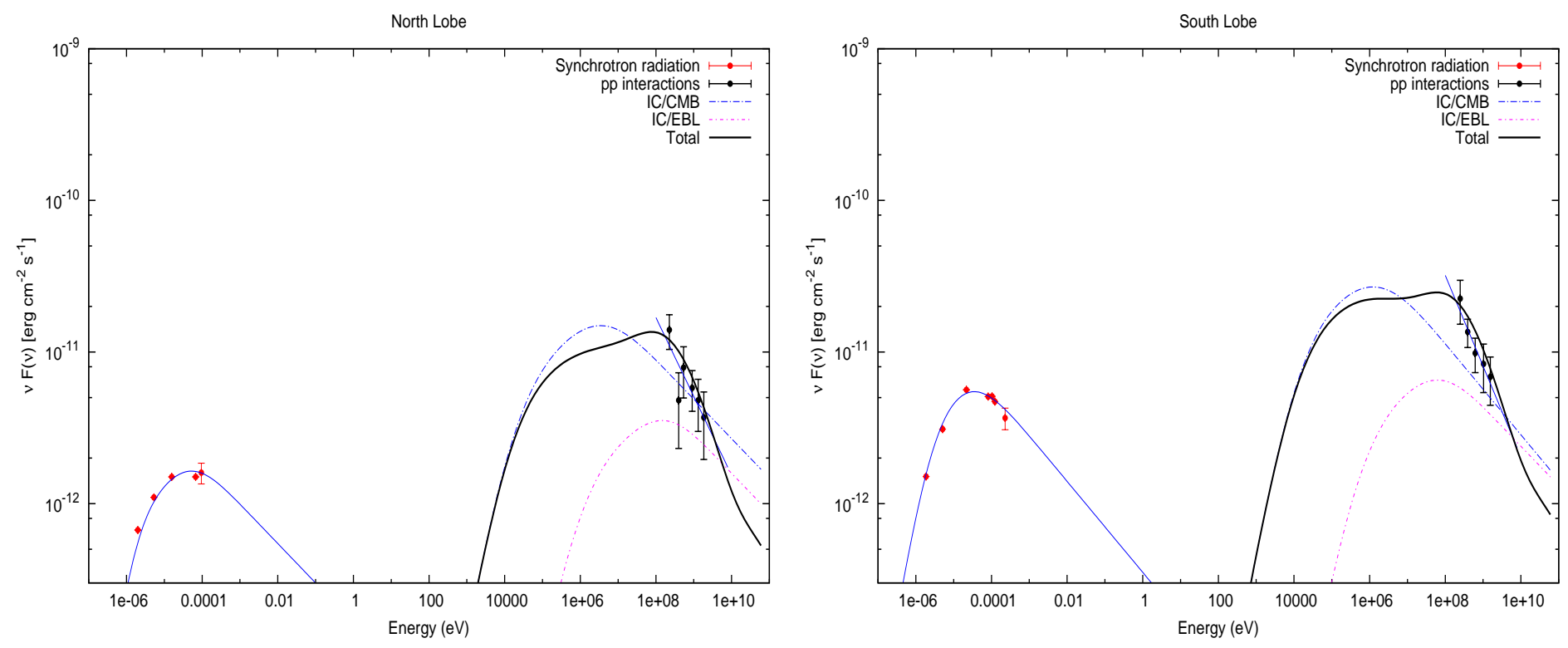

Fig. 3. - Fit of observed SED of the north (left) and south (right) lobes of Cen A. The component at higher energies is described through pp interaction and IC (CBM and EBL). IC emission is plotted with our model and the parameters obtained in table 1 and 2. 

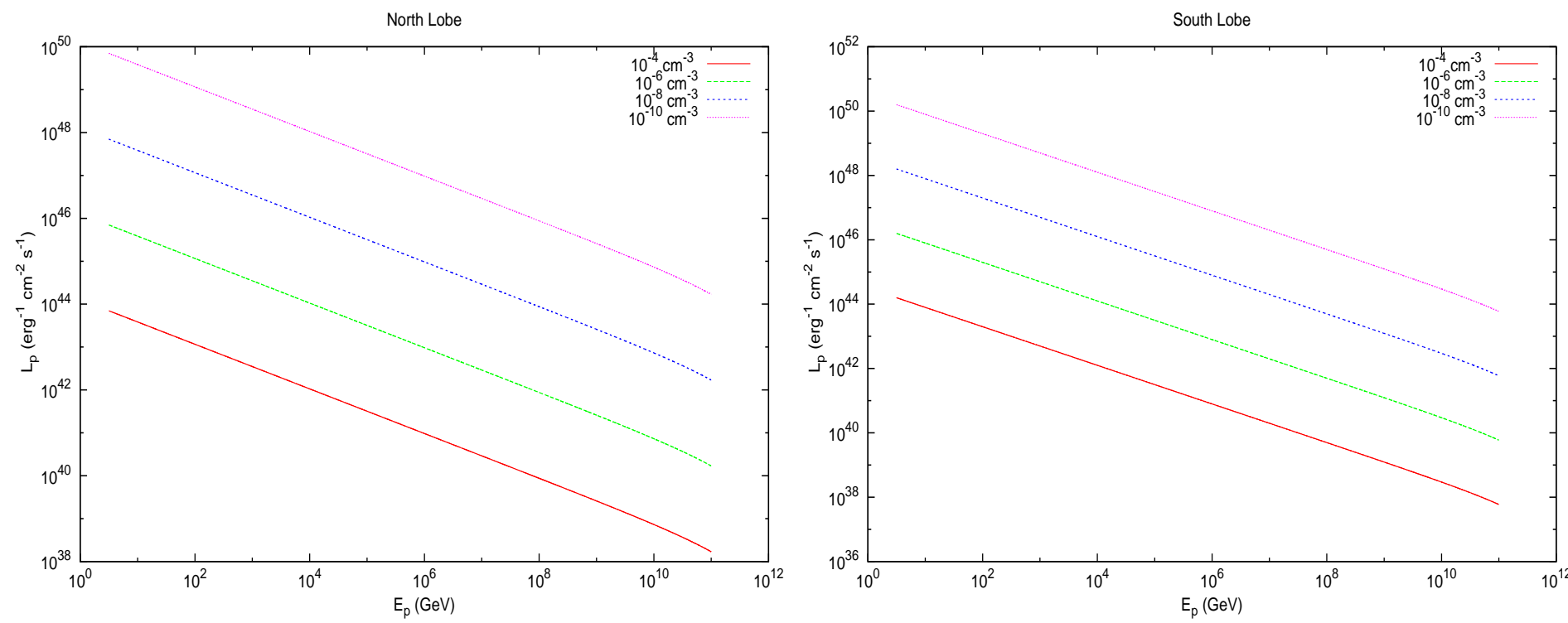

Fig. 4.- Proton luminosity $L_{p}$ as a function of energy $E_{p}$ for north (left) and south (right) lobes. These plots are generated as the thermal density range is $10^{-10} \mathrm{~cm}^{-3} \leq n_{p} \leq 10^{-4} \mathrm{~cm}^{-3}$. 

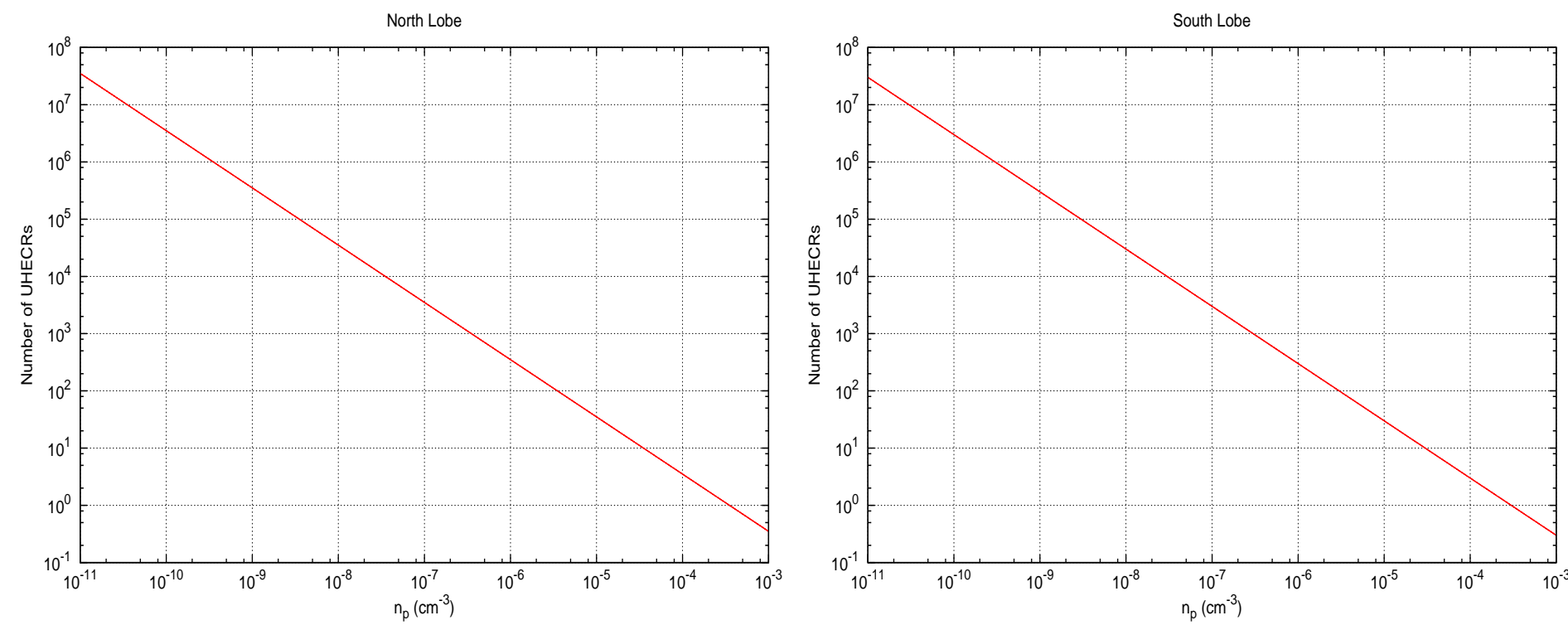

Fig. 5.- Number of UHECRs expected as a function of number density of thermal particles $\left(n_{p}\right)$ from the north (left) and south (right) lobes of Cen A. 

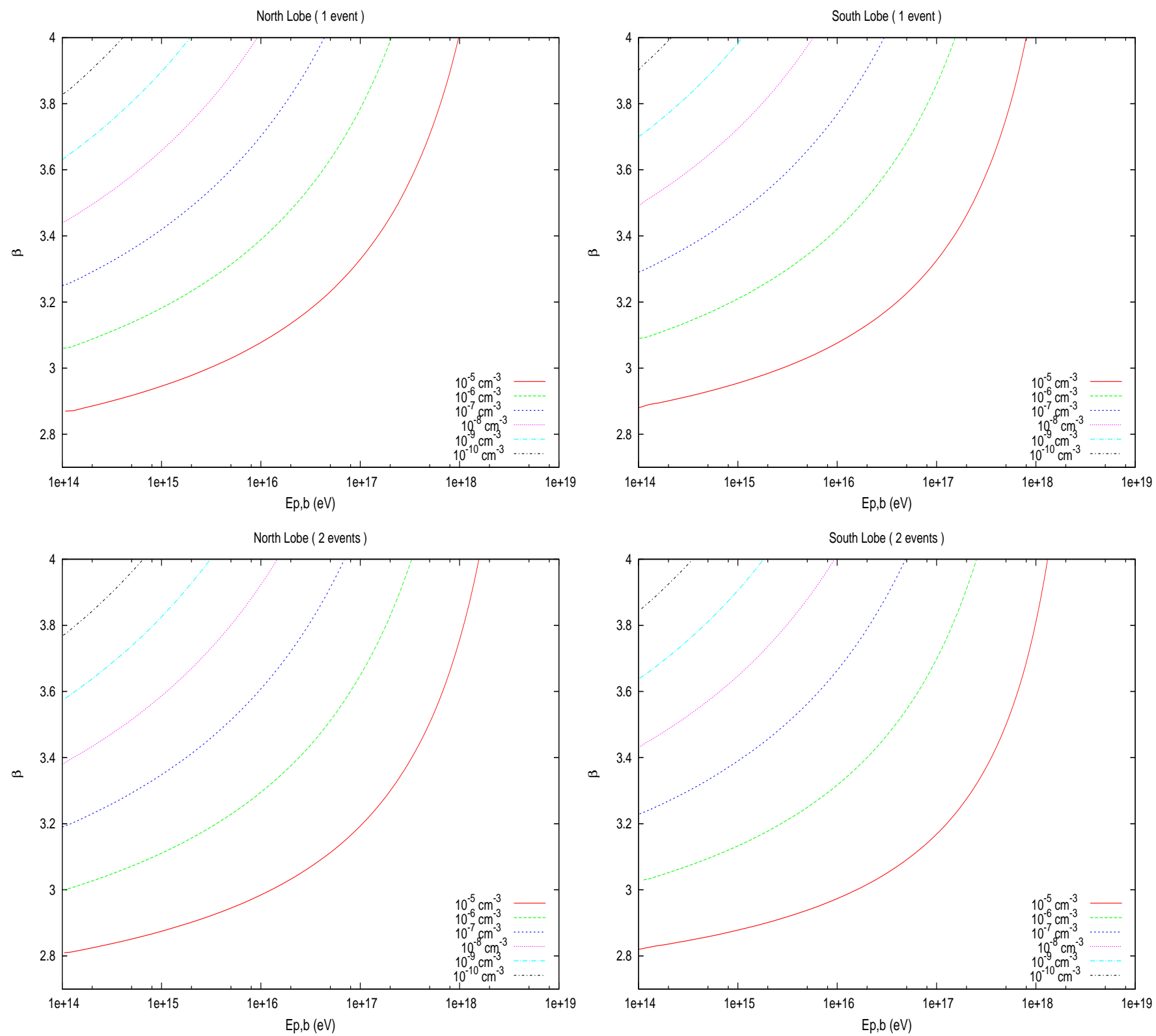

Fig. 6.- Contour plots of higher spectral index $(\beta)$ and break energy $\left(E_{p, b}\right)$ of the broken power law of accelerated protons (eq. 26) for which PAO would expect one (above) and two (below) events from the north (left) and south (right) lobes, when the number density of thermal particles is $10^{-10} \mathrm{~cm}^{-3} \leq n_{p} \leq 10^{-5} \mathrm{~cm}^{-3}$. 

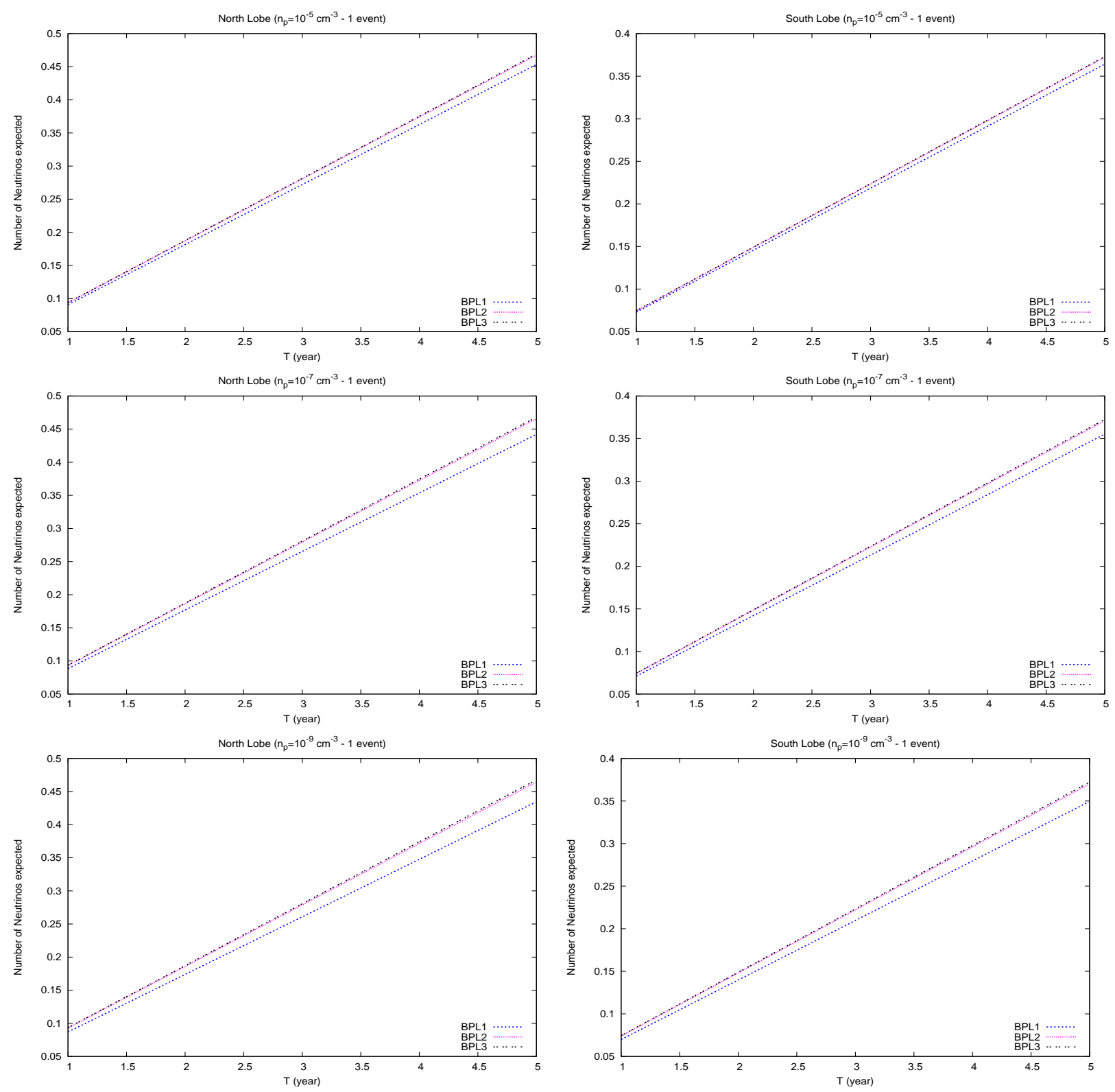

Fig. 7.- Number of neutrinos expected on IceCube when the neutrino threshold energy $\left(E_{\nu, t h}\right)$ is $1 \mathrm{TeV}$. These neutrinos are generated by pp interactions and normalized through $\gamma$-ray from north (left) and south (right) lobes. These figures show the number of neutrinos produced taking into account the parameters $\beta$ and $E_{p, b}$ of the broken power law of accelerated protons for which one UCHER would arrive in PAO. We have used the values of parameters $\beta$ and $E_{\nu, b}$ given in tables $\mathrm{B} 1$ and $\mathrm{B} 2$. 

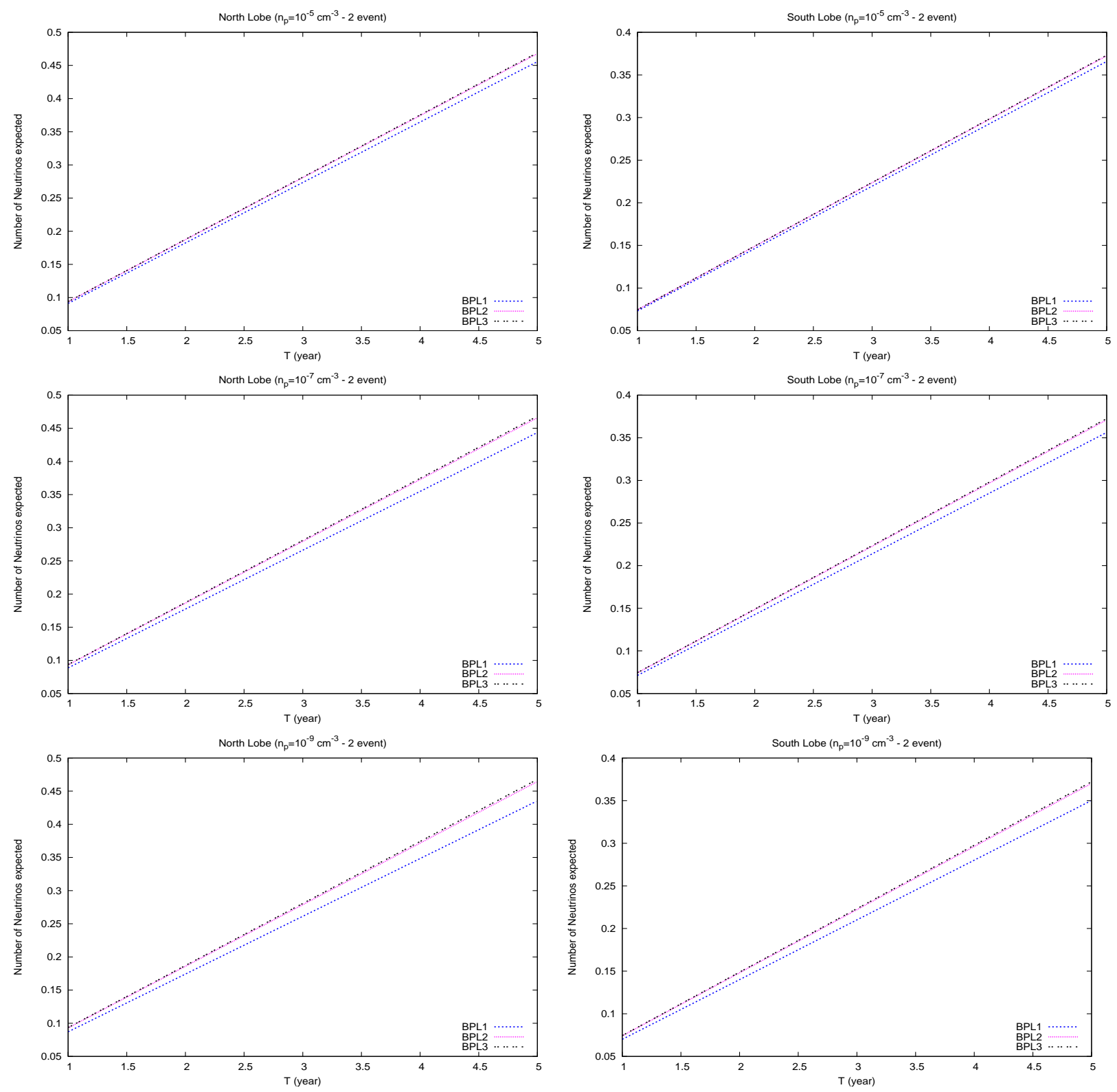

Fig. 8.- Number of neutrinos expected on IceCube when the neutrino threshold energy $\left(E_{\nu, t h}\right)$ is $1 \mathrm{TeV}$. These neutrinos are generated by pp interactions and normalized through $\gamma$-ray from north (left) and south (right) lobes. These figures show the number of neutrinos produced taking into account the parameters $\beta$ and $E_{p, b}$ of the broken power law of accelerated protons for which two UCHERs would arrive in PAO. We have used the values of parameters $\beta$ and $E_{\nu, b}$ given in tables $\mathrm{B} 1$ and $\mathrm{B} 2$. 


\section{A. Chi-square minimization}

Firstly, from pp interaction model (eq. 13), we fit the $\gamma$-ray spectrum using two parameters, the proportionality constant of pp spectrum $A_{p p, \gamma}$ (eq. 14) and the spectral index $\alpha$, as follow.

$$
\left(E_{\gamma}^{2} \frac{d N_{\gamma}}{d E_{\gamma}}\right)_{\pi^{0}}^{o b s}=[0]\left(\frac{E_{\gamma, \pi^{0}}^{o b s}}{\mathrm{GeV}}\right)^{2-[1]}
$$

After fitting we obtained the values

\begin{tabular}{lcccc}
\hline \hline Lobes & & & North & South \\
\hline \hline & Parameter & Symbol & Value & Value \\
\hline \hline Proportionality constant $\left(10^{-12} \mathrm{erg} / \mathrm{cm}^{2} / \mathrm{s}\right)$ & {$[0]$} & $A_{p p, \gamma}$ & $5.10 \pm 0.96$ & $8.07 \pm 1.58$ \\
Spectral index & {$[1]$} & $\alpha$ & $2.519 \pm 0.225$ & $2.598 \pm 0.254$ \\
Chi-square/NDF & & $\chi^{2} / \mathrm{NDF}$ & $2.748 / 4.0$ & $0.555 / 3.0$ \\
\hline
\end{tabular}

Table A1. The best fit of the set of pp interaction parameters obtained after fitting the $\gamma$-ray spectrum of north and south lobes.

Secondly, from synchrotron emission model (eq. 5) and the value of $\alpha$ (table 1), we fit the peak at radio wavelength using three parameters, the proportionality constant of synchrotron $A_{s y n, \gamma}$ (eq. 6), the characteristic and cut-off photon energies $\epsilon_{\gamma, m}^{o b s}$ and $\epsilon_{\gamma, c}^{o b s}$ (eq. 3), respectively as follow

$$
\epsilon_{\gamma}^{2} N_{\gamma}\left(\epsilon_{\gamma}\right)=[0] \begin{cases}\left(\frac{\epsilon_{\gamma}}{[2]}\right)^{4 / 3} & \epsilon_{\gamma}^{o b s}<[2], \\ \left(\frac{\epsilon_{\gamma}}{[2]}\right)^{-(\alpha-3) / 2} & {[2]<\epsilon_{\gamma}^{o b s}<[1],} \\ \left(\frac{[1]}{[2]}\right)^{-(\alpha-3) / 2}\left(\frac{\epsilon_{\gamma}}{[1]}\right)^{-(\alpha-2) / 2}, & {[1]<\epsilon_{\gamma}^{o b s}}\end{cases}
$$

\begin{tabular}{|c|c|c|c|c|}
\hline Lobes & & North & South & \\
\hline & Parameter & Symbol & Value & Value \\
\hline Proportionality constant $\left(10^{-12} \mathrm{erg} / \mathrm{cm}^{2} / \mathrm{s}\right)$ & [0] & $A_{s y n, \gamma}$ & $1.08 \pm 0.30$ & $4.51 \pm 0.64$ \\
\hline Cut-off photon energy $\left(10^{-5} \mathrm{eV}\right)$ & [1] & $\epsilon_{\gamma, c}^{o b s}$ & $5.703 \pm 0.557$ & $4.01 \pm 0.71$ \\
\hline Characteristic photon energy $\left(10^{-6} \mathrm{eV}\right)$ & [2] & $\epsilon_{\gamma, m}^{o b s}$ & $2.63 \pm 0.03$ & $3.48 \pm 1.70$ \\
\hline Chi-square/NDF & & $\chi^{2} / \mathrm{NDF}$ & $0.8616 / 2.0$ & $1.290 / 4.0$ \\
\hline
\end{tabular}

The values of the parameters obtained after fitting

Table A2. The best fit of the set of synchrotron parameters obtained after fitting spectrum at low energies of north and south lobes. 


\section{B. Neutrinos broken power law}

\begin{tabular}{|c|c|c|c|c|c|c|}
\hline & & one & event & & two & events \\
\hline & $\beta$ & $E_{\nu, b}(\mathrm{eV})$ & $N_{e v} / T(\text { year })^{-1}$ & $\beta$ & $E_{\nu, b}(\mathrm{eV})$ & $N_{e v} / T(\text { year })^{-1}$ \\
\hline \multicolumn{7}{|c|}{$n_{p}=10^{-5} \mathrm{~cm}^{-3}$} \\
\hline BPL1 & 2.87 & $0.5 \times 10^{13}$ & $9.07 \times 10^{-2}$ & 2.81 & $0.5 \times 10^{13}$ & $9.11 \times 10^{-2}$ \\
\hline BPL2 & 2.92 & $2.5 \times 10^{13}$ & $9.35 \times 10^{-2}$ & 2.85 & $2.5 \times 10^{13}$ & $9.36 \times 10^{-2}$ \\
\hline BPL3 & 2.95 & $0.5 \times 10^{14}$ & $9.38 \times 10^{-2}$ & 2.88 & $0.5 \times 10^{14}$ & $9.38 \times 10^{-2}$ \\
\hline \multicolumn{7}{|c|}{$n_{p}=10^{-6} \mathrm{~cm}^{-3}$} \\
\hline BPL1 & 3.06 & $0.5 \times 10^{13}$ & $8.94 \times 10^{-2}$ & 3.00 & $0.5 \times 10^{13}$ & $8.98 \times 10^{-2}$ \\
\hline BPL2 & 3.14 & $2.5 \times 10^{13}$ & $9.33 \times 10^{-2}$ & 3.07 & $2.5 \times 10^{13}$ & $9.33 \times 10^{-2}$ \\
\hline BPL3 & 3.18 & $0.5 \times 10^{14}$ & $9.37 \times 10^{-2}$ & 3.11 & $0.5 \times 10^{14}$ & $9.37 \times 10^{-2}$ \\
\hline \multicolumn{7}{|c|}{$n_{p}=10^{-7} \mathrm{~cm}^{-3}$} \\
\hline BPL1 & 3.25 & $0.5 \times 10^{13}$ & $8.84 \times 10^{-2}$ & 3.19 & $0.5 \times 10^{13}$ & $8.87 \times 10^{-2}$ \\
\hline BPL2 & 3.36 & $2.5 \times 10^{13}$ & $9.31 \times 10^{-2}$ & 3.29 & $2.5 \times 10^{13}$ & $9.32 \times 10^{-2}$ \\
\hline BPL3 & 3.42 & $0.5 \times 10^{14}$ & $9.37 \times 10^{-2}$ & 3.35 & $0.5 \times 10^{14}$ & $9.36 \times 10^{-2}$ \\
\hline \multicolumn{7}{|c|}{$n_{p}=10^{-8} \mathrm{~cm}^{-3}$} \\
\hline BPL1 & 3.44 & $0.5 \times 10^{13}$ & $8.76 \times 10^{-2}$ & 3.38 & $0.5 \times 10^{13}$ & $8.78 \times 10^{-2}$ \\
\hline BPL2 & 3.58 & $2.5 \times 10^{13}$ & $9.30 \times 10^{-2}$ & 3.51 & $2.5 \times 10^{13}$ & $9.30 \times 10^{-2}$ \\
\hline BPL3 & 3.66 & $0.5 \times 10^{14}$ & $9.36 \times 10^{-2}$ & 3.59 & $0.5 \times 10^{14}$ & $9.35 \times 10^{-2}$ \\
\hline \multicolumn{7}{|c|}{$n_{p}=10^{-9} \mathrm{~cm}^{-3}$} \\
\hline BPL1 & 3.63 & $0.5 \times 10^{13}$ & $8.69 \times 10^{-2}$ & 3.58 & $0.5 \times 10^{13}$ & $8.71 \times 10^{-2}$ \\
\hline BPL2 & 3.80 & $2.5 \times 10^{13}$ & $9.29 \times 10^{-2}$ & 3.74 & $2.5 \times 10^{13}$ & $9.29 \times 10^{-2}$ \\
\hline BPL3 & 3.90 & $0.5 \times 10^{14}$ & $9.35 \times 10^{-2}$ & 3.83 & $0.5 \times 10^{14}$ & $9.35 \times 10^{-2}$ \\
\hline \multicolumn{7}{|c|}{$n_{p}=10^{-10} \mathrm{~cm}^{-3}$} \\
\hline BPL1 & 3.83 & $0.5 \times 10^{13}$ & $8.63 \times 10^{-2}$ & 3.77 & $0.5 \times 10^{13}$ & $8.65 \times 10^{-2}$ \\
\hline BPL2 & 4.03 & $2.5 \times 10^{13}$ & $9.28 \times 10^{-2}$ & 3.96 & $2.5 \times 10^{13}$ & $9.28 \times 10^{-2}$ \\
\hline BPL3 & 4.14 & $0.5 \times 10^{14}$ & $9.35 \times 10^{-2}$ & 4.07 & $0.5 \times 10^{14}$ & $9.35 \times 10^{-2}$ \\
\hline
\end{tabular}

Table B1. Number of neutrinos above 1 TeV expected per year from the north lobe (see fig. 3 of the left). Here we show the parameters $(\beta$ and $E_{\nu, b}$ ) of neutrino spectrum (eq. 36) for three broken power laws (BPLs) when one and two UCHERs are expected in PAO. 


\begin{tabular}{|c|c|c|c|c|c|c|}
\hline & & one & event & & two & events \\
\hline & $\beta$ & $E_{\nu, b}(\mathrm{eV})$ & $N_{e v} / T(\text { year })^{-1}$ & $\beta$ & $E_{\nu, b}(\mathrm{eV})$ & $N_{e v} / T(\text { year })^{-1}$ \\
\hline \multicolumn{7}{|c|}{$n_{p}=10^{-5} \mathrm{~cm}^{-3}$} \\
\hline BPL1 & 2.88 & $0.5 \times 10^{13}$ & $7.28 \times 10^{-2}$ & 2.82 & $0.5 \times 10^{13}$ & $7.32 \times 10^{-2}$ \\
\hline BPL2 & 2.93 & $2.5 \times 10^{13}$ & $7.44 \times 10^{-2}$ & 2.86 & $2.5 \times 10^{13}$ & $7.45 \times 10^{-2}$ \\
\hline BPL3 & 2.95 & $0.5 \times 10^{14}$ & $7.46 \times 10^{-2}$ & 2.88 & $0.5 \times 10^{14}$ & $7.46 \times 10^{-2}$ \\
\hline \multicolumn{7}{|c|}{$n_{p}=10^{-6} \mathrm{~cm}^{-3}$} \\
\hline BPL1 & 3.09 & $0.5 \times 10^{13}$ & $7.18 \times 10^{-2}$ & 3.03 & $0.5 \times 10^{13}$ & $7.21 \times 10^{-2}$ \\
\hline BPL2 & 3.17 & $2.5 \times 10^{13}$ & $7.43 \times 10^{-2}$ & 3.09 & $2.5 \times 10^{13}$ & $7.43 \times 10^{-2}$ \\
\hline BPL3 & 3.21 & $0.5 \times 10^{14}$ & $7.45 \times 10^{-2}$ & 3.13 & $0.5 \times 10^{14}$ & $7.46 \times 10^{-2}$ \\
\hline \multicolumn{7}{|c|}{$n_{p}=10^{-7} \mathrm{~cm}^{-3}$} \\
\hline BPL1 & 3.29 & $0.5 \times 10^{13}$ & $7.10 \times 10^{-2}$ & 3.23 & $0.5 \times 10^{13}$ & $7.13 \times 10^{-2}$ \\
\hline BPL2 & 3.40 & $2.5 \times 10^{13}$ & $7.42 \times 10^{-2}$ & 3.33 & $2.5 \times 10^{13}$ & $7.42 \times 10^{-2}$ \\
\hline BPL3 & 3.47 & $0.5 \times 10^{14}$ & $7.45 \times 10^{-2}$ & 3.39 & $0.5 \times 10^{14}$ & $7.45 \times 10^{-2}$ \\
\hline \multicolumn{7}{|c|}{$n_{p}=10^{-8} \mathrm{~cm}^{-3}$} \\
\hline BPL1 & 3.49 & $0.5 \times 10^{13}$ & $7.04 \times 10^{-2}$ & 3.43 & $0.5 \times 10^{13}$ & $7.06 \times 10^{-2}$ \\
\hline BPL2 & 3.64 & $2.5 \times 10^{13}$ & $7.41 \times 10^{-2}$ & 3.57 & $2.5 \times 10^{13}$ & $7.41 \times 10^{-2}$ \\
\hline BPL3 & 3.72 & $0.5 \times 10^{14}$ & $7.45 \times 10^{-2}$ & 3.65 & $0.5 \times 10^{14}$ & $9.45 \times 10^{-2}$ \\
\hline \multicolumn{7}{|c|}{$n_{p}=10^{-9} \mathrm{~cm}^{-3}$} \\
\hline BPL1 & 3.70 & $0.5 \times 10^{13}$ & $6.99 \times 10^{-2}$ & 3.64 & $0.5 \times 10^{13}$ & $7.00 \times 10^{-2}$ \\
\hline BPL2 & 3.88 & $2.5 \times 10^{13}$ & $7.40 \times 10^{-2}$ & 3.81 & $2.5 \times 10^{13}$ & $7.40 \times 10^{-2}$ \\
\hline BPL3 & 3.98 & $0.5 \times 10^{14}$ & $7.44 \times 10^{-2}$ & 3.91 & $0.5 \times 10^{14}$ & $7.44 \times 10^{-2}$ \\
\hline \multicolumn{7}{|c|}{$n_{p}=10^{-10} \mathrm{~cm}^{-3}$} \\
\hline BPL1 & 3.90 & $0.5 \times 10^{13}$ & $6.95 \times 10^{-2}$ & 3.85 & $0.5 \times 10^{13}$ & $6.96 \times 10^{-2}$ \\
\hline BPL2 & 4.12 & $2.5 \times 10^{13}$ & $7.40 \times 10^{-2}$ & 4.05 & $2.5 \times 10^{13}$ & $7.40 \times 10^{-2}$ \\
\hline BPL3 & 4.24 & $0.5 \times 10^{14}$ & $7.44 \times 10^{-2}$ & 4.17 & $0.5 \times 10^{14}$ & $7.44 \times 10^{-2}$ \\
\hline
\end{tabular}

Table B2. Number of neutrinos above $1 \mathrm{TeV}$ expected per year from the south lobe (see fig. 3 of the left). Here we show the parameters $(\beta$ and $E_{\nu, b}$ ) of neutrino spectrum (eq. 36 for three broken power laws (BPLs) when one and two UCHERs are expected in PAO. 